\title{
Methionine adenosyltransferase 1a antisense oligonucleotides activate the liver-brown adipose tissue axis preventing obesity and associated hepatosteatosis
}

\author{
Diego Sáenz de Urturi ${ }^{1}$, Xabier Buqué (1) 1,2, Begoña Porteiro ${ }^{3}$, Cintia Folgueira (1) ${ }^{4}$, Alfonso Mora (1) ${ }^{4}$, \\ Teresa C. Delgado (1) ${ }^{5}$, Endika Prieto-Fernández (10 ${ }^{6}$, Paula Olaizola (1) ${ }^{7}$, Beatriz Gómez-Santos ${ }^{1}$, \\ Maider Apodaka-Biguri', Francisco González-Romero', Ane Nieva-Zuluaga', Mikel Ruiz de Gauna', \\ Naroa Goikoetxea-Usandizaga (i) ${ }^{5}$, Juan Luis García-Rodríguez (10) 1, Virginia Gutierrez de Juan', \\ Igor Aurrekoetxea1,2, Valle Montalvo-Romeral ${ }^{4}$, Eva M. Novoa ${ }^{3}$, Idoia Martín-Guerrero (1) 6, \\ Marta Varela-Rey (10 5,8,9, Sanjay Bhanot ${ }^{10}$, Richard Lee (iD ${ }^{10}$, Jesus M. Banales ${ }^{7,8,11,12}$, Wing-Kin Syn ${ }^{1,13,14}$,

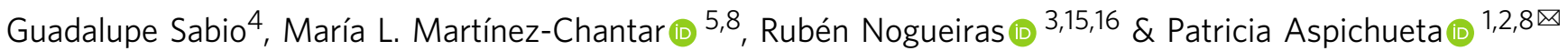

\begin{abstract}
Altered methionine metabolism is associated with weight gain in obesity. The methionine adenosyltransferase (MAT), catalyzing the first reaction of the methionine cycle, plays an important role regulating lipid metabolism. However, its role in obesity, when a plethora of metabolic diseases occurs, is still unknown. By using antisense oligonucleotides (ASO) and genetic depletion of Mat1a, here, we demonstrate that Mat1a deficiency in diet-induce obese or genetically obese mice prevented and reversed obesity and obesity-associated insulin resistance and hepatosteatosis by increasing energy expenditure in a hepatocyte FGF21 dependent fashion. The increased NRF2-mediated FGF21 secretion induced by targeting Mat1a, mobilized plasma lipids towards the BAT to be catabolized, induced thermogenesis and reduced body weight, inhibiting hepatic de novo lipogenesis. The beneficial effects of Mat1a ASO were abolished following FGF21 depletion in hepatocytes. Thus, targeting Mat1a activates the liver-BAT axis by increasing NRF2mediated FGF21 secretion, which prevents obesity, insulin resistance and hepatosteatosis.
\end{abstract}

\footnotetext{
${ }^{1}$ Department of Physiology, Faculty of Medicine and Nursing, University of the Basque Country UPV/EHU, Leioa, Spain. ${ }^{2}$ Biocruces Bizkaia Health Research Institute, Barakaldo, Spain. ${ }^{3}$ Department of Physiology, CIMUS, University of Santiago de Compostela-Instituto de Investigación Sanitaria, Santiago de Compostela, Spain. ${ }^{4}$ Myocardial Pathophysiology, Centro Nacional de Investigaciones Cardiovasculares (CNIC), Madrid, Spain. ${ }^{5}$ Liver Disease Laboratory, CIC bioGUNE-BRTA (Basque Research \& Technology Alliance), Derio, Spain. ${ }^{6}$ Department of Genetics, Physical Anthropology and Animal Physiology, Faculty of Science and Technology, University of the Basque Country UPV/EHU, Leioa, Spain. ${ }^{7}$ Department of Liver and Gastrointestinal Diseases, Biodonostia Health Research Institute-Donostia University Hospital, University of the Basque Country (UPV/EHU), San Sebastian, Spain. ${ }^{8}$ National Institute for the Study of Liver and Gastrointestinal Diseases (CIBERehd, Instituto de Salud Carlos III), Madrid, Spain. ${ }^{9}$ Gene Regulatory Control in Disease Laboratory, CIMUS, University of Santiago de Compostela-Instituto de Investigación Sanitaria, Santiago de Compostela, Spain. ${ }^{10}$ IONIS Pharmaceuticals, Carlsbad, CA USA. ${ }^{11}$ Ikerbasque, Basque Foundation for Science, Bilbao, Spain. ${ }^{12}$ Department of Biochemistry and Genetics, School of Sciences, University of Navarra, Pamplona, Spain. ${ }^{13}$ Section of Gastroenterology, Ralph H Johnson, VAMC, Charleston, SC, USA. ${ }^{14}$ Division of Gastroenterology and Hepatology, Medical University of South Carolina, Charleston, SC, USA. ${ }^{15}$ CIBER Fisiopatología de la Obesidad y Nutrición (CIBERobn), Santiago de Compostela, Spain. ${ }^{16}$ Galician

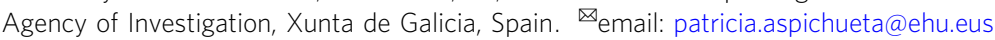


ncreased prevalence of obesity has led to a tsunami of metabolic diseases such as type 2 diabetes mellitus, dyslipidemia, and nonalcoholic fatty liver disease (NAFLD) ${ }^{1}$.

NAFLD is the most common cause of chronic liver disease in Western countries ${ }^{2}$. It ranges from hepatosteatosis to nonalcoholic steatohepatitis (NASH), a more advanced form of the disease. There are still no specific pharmacological treatments for NAFLD but as obesity is a major risk factor, treatments that impact body weight and glucose control have been investigated ${ }^{3}$.

In patients with obesity, where NAFLD prevalence is $\sim 80 \%$, serum levels of S-adenosylmethionine (SAMe) are elevated and correlate with abdominal adiposity, fat mass and higher calorie intake, suggesting the increased synthesis of liver SAMe ${ }^{4,5}$. SAMe is synthesized in the first reaction of the methionine cycle through the action of methionine adenosyltransferase (MAT), which uses methionine and $\mathrm{ATP}^{6}$. Obesity development associates with high methionine intake ${ }^{7,8}$, which in turn, produces an increase of serum SAMe levels ${ }^{9}$. In concordance, dietary methionine restriction (MR) reduces adiposity and leptin levels, increases adiponectin release and recovers insulin sensitivity ${ }^{10,11}$.

Deficiency in several enzymes involved in the methionine cycle confers resistance to obesity and the associated co-morbidities. In mice, lack of phosphatidylethanolamine N-methyltransferase (PEMT) prevents high-fat diet (HFD)-induced obesity and insulin resistance by promoting energy expenditure ${ }^{12,13}$. Nicotinamide N-methyltransferase (NNMT) regulates glucose and cholesterol metabolism ${ }^{14}$; however, knockdown of $\mathrm{Nnmt}$ also induces energy consumption and protects mice from obesity and hepatosteatosis ${ }^{15}$.

Chronic changes in liver SAMe levels in lean mice have been associated with the onset and progression of NAFLD with age. Accumulation of liver SAMe due to its decreased catabolism in glycine N-methyltransferase (Gnmt)-knockout $(\mathrm{KO})$ mice results in the spontaneous development of NAFLD and hepatocarcinogenesis with increasing age ${ }^{16,17}$. Curiously, chronic low liver SAMe levels in mice due to the absence of methionine adenosyltransferase 1a (Mat1a), which is mainly expressed in liver and encodes MATa1, that oligomerizes to generate MATI/III ${ }^{6,18}$, also leads to the spontaneous onset of NASH with age ${ }^{6,19}$. Thus, maintaining physiological levels of liver SAMe in an appropriate range is essential to fine-tune liver function during aging.

Here, we investigated if the pharmacological (antisense oligonucleotide (ASO) treatment) knockdown of the Mat1a gene, which is involved in the first reaction of the methionine cycle, provides beneficial outcomes in diet- and genetically- induced obesity and obesity-related NAFLD. We designed two different ASO to knockdown Mat1a, and used diet-induced obese, $o b / o b$, and hepatocyte-specific fibroblast growth factor (Fgf)21-KO mice. Mat1a-KO mice were also used. Metabolic fluxes using radiolabeled substrates, very-low density lipoprotein (VLDL) secretion and dietary lipid metabolism were analyzed. The results showed that targeting Mat1a prevents and reverses obesity as well as obesity-associated dyslipidemia, insulin resistance and hepatosteatosis. Studies performed in vivo and in vitro showed that the hepatocyte secretion of FGF21 mediates increased thermogenesis of brown adipose tissue (BAT) and that FGF21 is regulated, in part, by nuclear factor erythroid 2-related factor 2 (NRF2).

\section{Results}

Targeting Mat1a reverses diet-induced obesity (DIO). Several changes in methionine cycle are linked to protection against obesity in mice $11,12,15$. Therefore, we investigated if targeting Mat1a could improve and/or prevent obesity and the associated whole-body metabolic dysregulation. Mat1a knockdown (KD) was performed using two different Mat1a ASO (Mat1a ASO and Mat1a ASO2). The results showed that administration of both Mat1a ASO or Mat1a ASO2 did not induce changes in liver or renal damage markers in HFD-fed mice (Table 1 and Supplementary Table 1). Treatment with Mat1a ASO or Mat1a ASO2 led to a $90-100 \%$ downregulation of Mat1a in HFD-fed mice liver (Supplementary Fig. 1a, b). The results showed that feeding a HFD did not alter liver levels of MATI/III (Fig. 1a), or induce the transcription of Mat1a in BAT or white adipose tissue (WAT), in which protein levels were absent (Supplementary Fig. 1c). However, targeting Mat1a with ASO markedly reduced liver MATI/III levels (Fig. 1a) and induced a loss in body weight to that comparable with chow diet-fed (CD) mice (Fig. 1b, c and Supplementary Fig. 1d). Most body weight loss was due to the lower fat mass, although there was also a decrease in lean mass (Fig. 1d). Food intake (Fig. 1e) and rectal temperature (Supplementary Fig. 1e) were unchanged. The same profile was observed when HFD-fed mice were treated with Mat1a ASO2 (Supplementary Fig. 1f). Moreover, the HFD-induced impairment in glucose disposal, in the release of insulin in response to glucose (Fig. 1f), in fasting insulin levels (Supplementary Fig. 1g) and in insulin sensitivity (Fig. 1g) were prevented when HFD-fed mice were treated with the Mat1a ASO (Fig. 1f, g). Even more, the glucose tolerance test (GTT), the release of insulin and the insulin

Table 1 Corporal and hepatic and renal function parameters in ASO-treated mice.

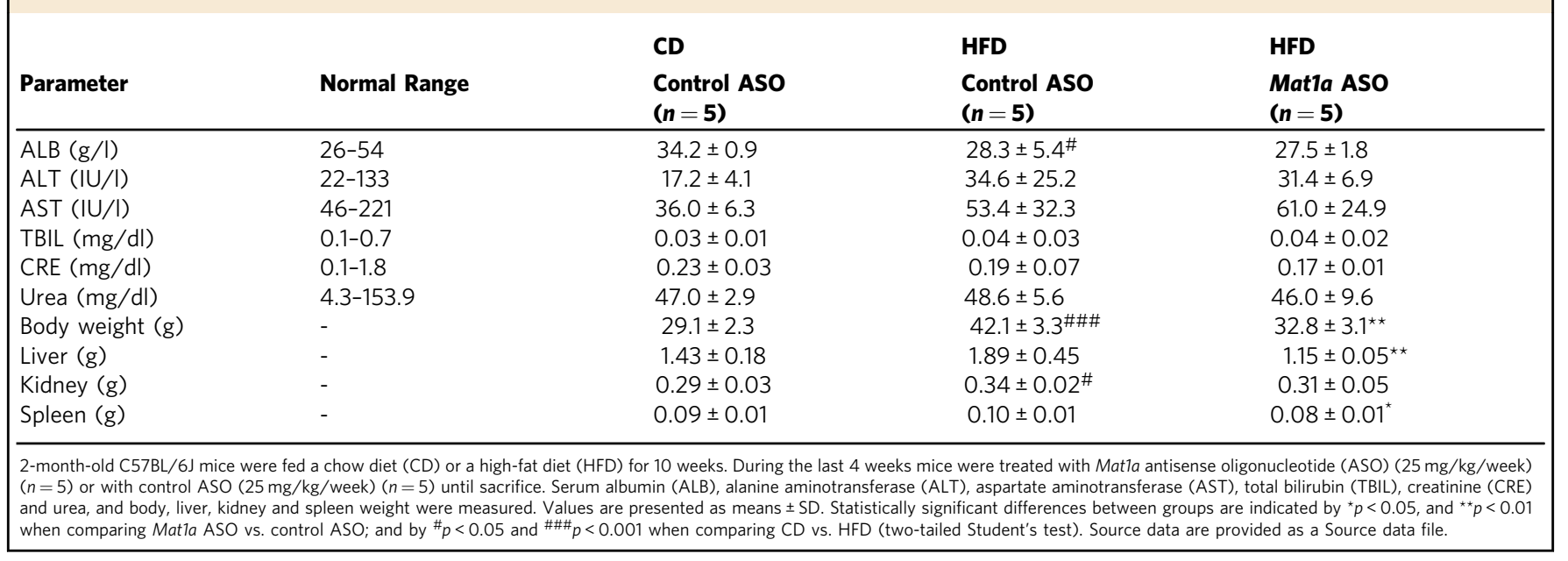



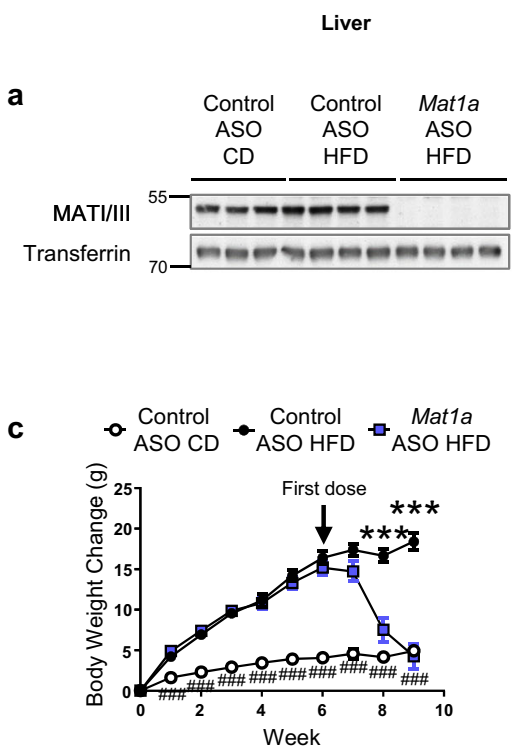

f

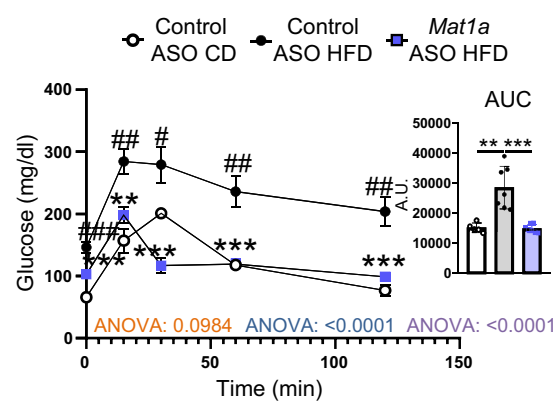

h
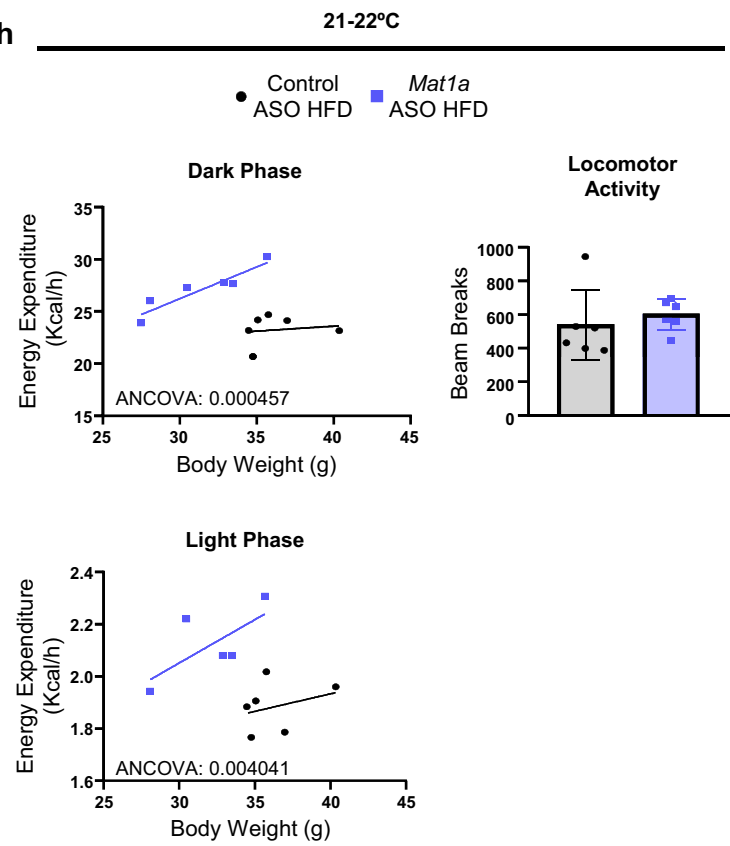

tolerance test (ITT) after the first dose of ASO, showed that targeting Mat1a, prevented the impairment in glucose disposal before the marked body weight loss (Supplementary Fig. 1h).

To investigate if the improved phenotype was a result of altered energy expenditure, metabolic studies were performed using an indirect calorimetric system. The analysis revealed that in HFD-fed

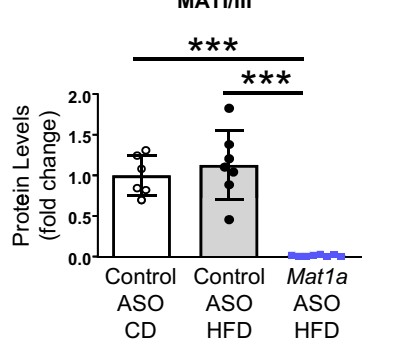

b

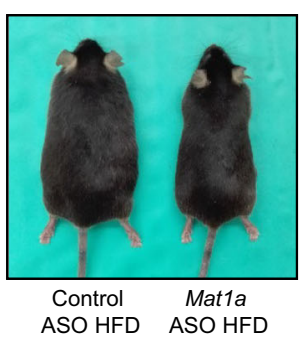

e

Food Intake

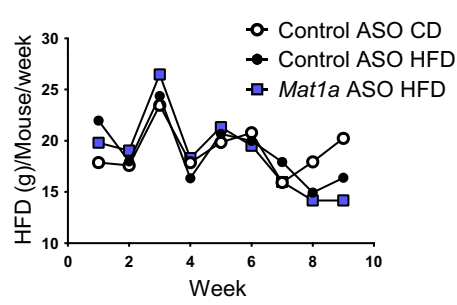

g

ITT
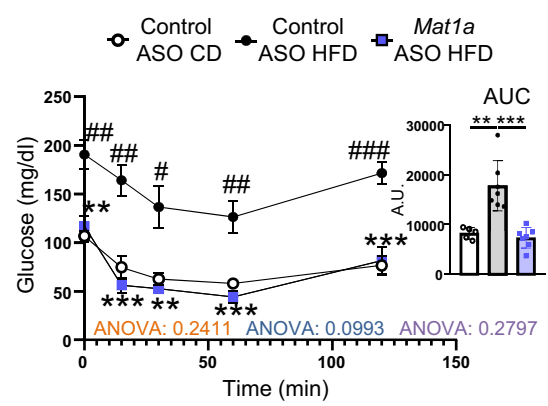

Thermoneutrality $30^{\circ} \mathrm{C}$
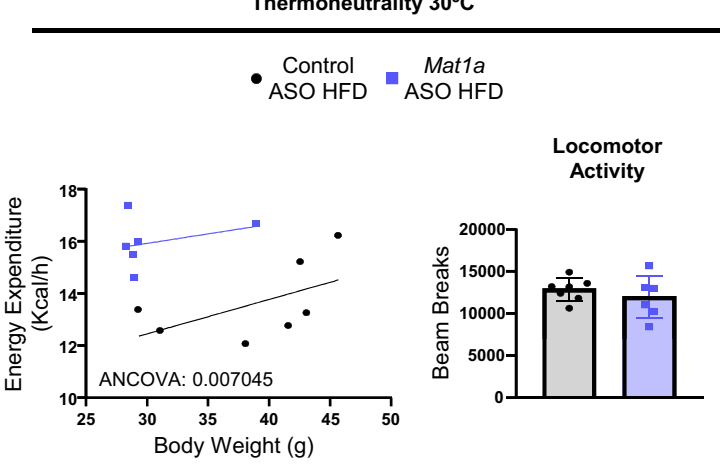

mice, after the four injections of treatment, Mat1a ASO induced increased energy expenditure with lower body weight and the same locomotor activity (Fig. 1h) and respiratory quotient (Supplementary Fig. 1i) when compared with control ASO treatment. The same profile was observed during the light period, when animals were inactive (Fig. 1h) and in thermoneutrality (Fig. 1i), a specific 
Fig. 1 Targeting Mat1a reverses diet-induced obesity. Two-month-old C57BL/6J mice were fed a chow-diet (CD) or a high-fat diet (HFD) for 10 weeks. During the last 4 weeks mice were treated with Mat1a antisense oligonucleotide (ASO) or control ASO ( $25 \mathrm{mg} / \mathrm{kg} /$ week) until sacrifice. a Knockdown of Mat1a ASO. MATI/III and transferrin blots and densitometries in liver of CD- $(n=6)$ and HFD-fed control $(n=7)$ and Mat1a $(n=8)$ ASO-treated mice. b Representative photograph of HFD-fed ASO-treated mice. c Body weight change for CD- $(n=6)$ and HFD-fed control $(n=7)$ and Mat7a $(n=8)$ ASOtreated mice. $\mathbf{d}$ Body composition in HFD-fed control $(n=7)$ and Mat7a $(n=7)$ ASO-treated mice. e Food intake for CD- $(n=2$ cages) and HFD-fed control ( $n=2$ cages) and Mat1a ( $n=2$ cages) ASO-treated mice. $\mathbf{f}$ Glucose tolerance test (GTT), insulin serum levels and $\mathbf{g}$ insulin tolerance test (ITT) of CD- $(n=5)$ and HFD-fed control $(n=7)$ and Mat1a $(n=7)$ ASO-treated mice at the third week of treatment. Data are also indicated as area under the curve (AUC) expressed in arbitrary units (A.U.). h Dark-phase energy balance, locomotor activity, and light phase energy balance in HFD-fed control ( $n=6$ in light and dark phase) and Mat1a ( $n=5$ in light and $n=6$ in dark phase) ASO-treated mice housed in metabolic cages over 2 days at the end of the treatment period. i Overall energy balance and locomotor activity in thermoneutrality in HFD-fed control $(n=7)$ and Mat1a $(n=6)$ ASO-treated mice housed in metabolic cages over 2 days at the end of the treatment period. Values are means \pm SEM for time course representations, and means \pm SD for histograms. Statistically significant differences are indicated by ${ }^{\star} p<0.05,{ }^{\star \star} p<0.01$, and ${ }^{{ }^{\star \star \star}} p<0.001$ for Control ASO HFD vs. Mat1a ASO HFD; and

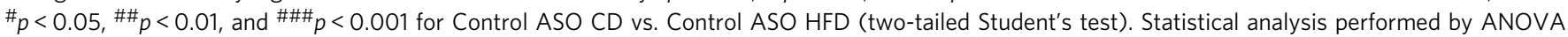
test comparing Control ASO CD vs. Control ASO HFD; Control ASO HFD vs. Mat1a ASO HFD; Control ASO CD vs. Mat7a ASO HFD is presented in GTT and ITT curves. Statistical analysis for energy expenditure was performed by two-way ANCOVA test. Source data are provided as a Source data file.

measurement of non-shivering thermogenesis. The long-term treatment with the Mat1a ASOs showed that after 7 injections of Mat1a ASO, the body weight reached the CD-fed mice body weight, and maintained stable (during the following 2 injections), with no changes in food intake (Supplementary Fig. 1j). These results are in concordance with the fact that targeting Mat1a in CDfed mice did not induce changes in body weight (Supplementary Table 2). The analysis of damage markers of liver and kidney function showed that the long-term treatment did not increase levels of these parameters above the normality range for mice. However, there was a slight increase in some parameters of liver function (Supplementary Table 3).

To assess if the chronic Mat1a deficiency could prevent the DIO and the associated insulin resistance, Mat1a-KO mice were fed a HFD for 10 weeks. Mat1a-KO mice were fully resistant to DIO, whereas the HFD-fed wild-type (WT) mice gained the expected weight (Supplementary Fig. 2a). Similarly, as occurred with mice treated with Mat1a ASOs, food intake maintained unaltered (Supplementary Fig. 2b) whereas the GTT and ITT showed that Mat1a-KO mice were protected from the HFDinduced glucose intolerance and insulin resistance (Supplementary Fig. 2c, d).

Mat1a ASOs induce thermogenesis in BAT. The results showed that targeting liver Mat1a increased energy expenditure even in thermoneutrality, specific of non-shivering thermogenesis. Therefore, we performed a detailed study in adipose tissue, and observed that Mat1a ASO reduced the HFD-induced BAT (Fig. 2a) and WAT adipocyte size (Supplementary Fig. 3a). HFDfed Mat1a ASO (Fig. 2b), Mat1a ASO2 (Supplementary Fig. 4a), and Mat1a KO mice (Supplementary Fig. 4a), all exhibited an increase in fatty acid oxidation (FAO) in BAT when compared to the corresponding controls. Targeting Mat1a also enhanced the oxygen consumption rate (OCR) in BAT mitochondria (Fig. 2c), which was associated with increased release of glycerol when lipolysis was measured ex vivo from freshly isolated BAT (Fig. 2d). However, release of fatty acids maintained unchanged probably because they are being used as substrate for the increased FAO in BAT (Fig. 2d). Inhibition of liver Mat1a led to increased interscapular temperature (Fig. 2e), increased uncoupling protein1 (UCP1) levels and S6 signaling, while no effects were observed in the mitogens activated protein kinase p38 or in protein kinase A (PKA) phosphorylation (Fig. 2f).

Similar to BAT, in WAT, the acid-soluble metabolites (ASM) release during FAO (Supplementary Fig. 3b) and lipolysis were elevated in Mat1a ASO-treated mice (Supplementary Fig. 3c). The increased lipolysis, coupled a decreased serum fatty acid levels (Supplementary Fig. 3d) and a tendency to increase UCP1 and pS6 signaling while no changes were observed in p38 or its phosphorylated form (Supplementary Fig. 3e).

Treatment with Mat1a ASO did not induce increases in serum epinephrine or norepinephrine levels (Fig. $2 \mathrm{~g}$ ) nor in BAT epinephrine levels (Supplementary Table 4). The increased expression of Ucpl induced by Matla ASO in BAT was associated with increased peroxisome proliferator-activated receptors (Ppar) alpha (a), Ppar gamma (g) and adiponectin $\mathrm{Q}$ (Adipoq) expression (Fig. 2h). In WAT, Mat1a ASO induced the increase in Adipoq expression while the expression of Pparg remained unaltered (Supplementary Fig. 3f). These changes were associated with increased serum adiponectin levels (Supplementary Fig. 3g).

Supporting the increased thermogenesis in BAT, the acute administration of a $\beta 3$ adrenergic agonist to Mat1a ASO-treated mice showed a higher consumption of $\mathrm{VO}_{2} 30 \mathrm{~min}$ and, more markedly, 45 min after the injection (Supplementary Fig 4b), and an increased response in the induction of the expression of Ucp1 and Ppargcla, both involved in thermogenesis (Supplementary Fig. 4c). This indicates an increased sympathetic response, which is essential for BAT non-shivering thermogenesis. Finally, to investigate if the effect in BAT was cell-autonomous or was more linked to a peripheral effect, FAO and the expression of Pparg (in differentiation) and Ucp1 (after the induction) were measured in BAT primary cells (Supplementary Fig. 4d, e). The results showed that BAT primary cells maintained in culture did not show the increased FAO or Ucp1 and Pparg expression induced in BAT by the in vivo treatment with Mat1a ASO, suggesting that these effects are not cell-autonomous of BAT adipocytes.

Finally, the results showed that targeting liver Mat1a downregulated Acaca expression (Supplementary Fig. 5a) and acetylCoA carboxylase (ACC) and fatty acid synthase (FAS) protein levels in BAT (Supplementary Fig. 5b), involved in the de novo lipogenesis, suggesting a contribution to the decreased lipid content in BAT.

Altogether, the results here demonstrate that targeting liver Mat1a, induces BAT thermogenesis, WAT lipolysis and the secretion of adiponectin. The results suggest that activation of S6 signaling might underline the induction of UCP1 in BAT, as has been demonstrated before ${ }^{20}$.

Mat1a ASOs protect from high-fat diet-induced NAFLD. The most effective treatments to reverse NAFLD are those that lead to weight $\operatorname{loss}^{21}$. Thus, we further investigated if the Mat1a ASO could also reduce the HFD-induced hepatosteatosis. The results showed that the HFD-induced liver storage of lipid droplets and increased triglyceride (TG) concentration (Fig. 3a) after 10 weeks of a HFD, were prevented when HFD-fed mice were treated with 

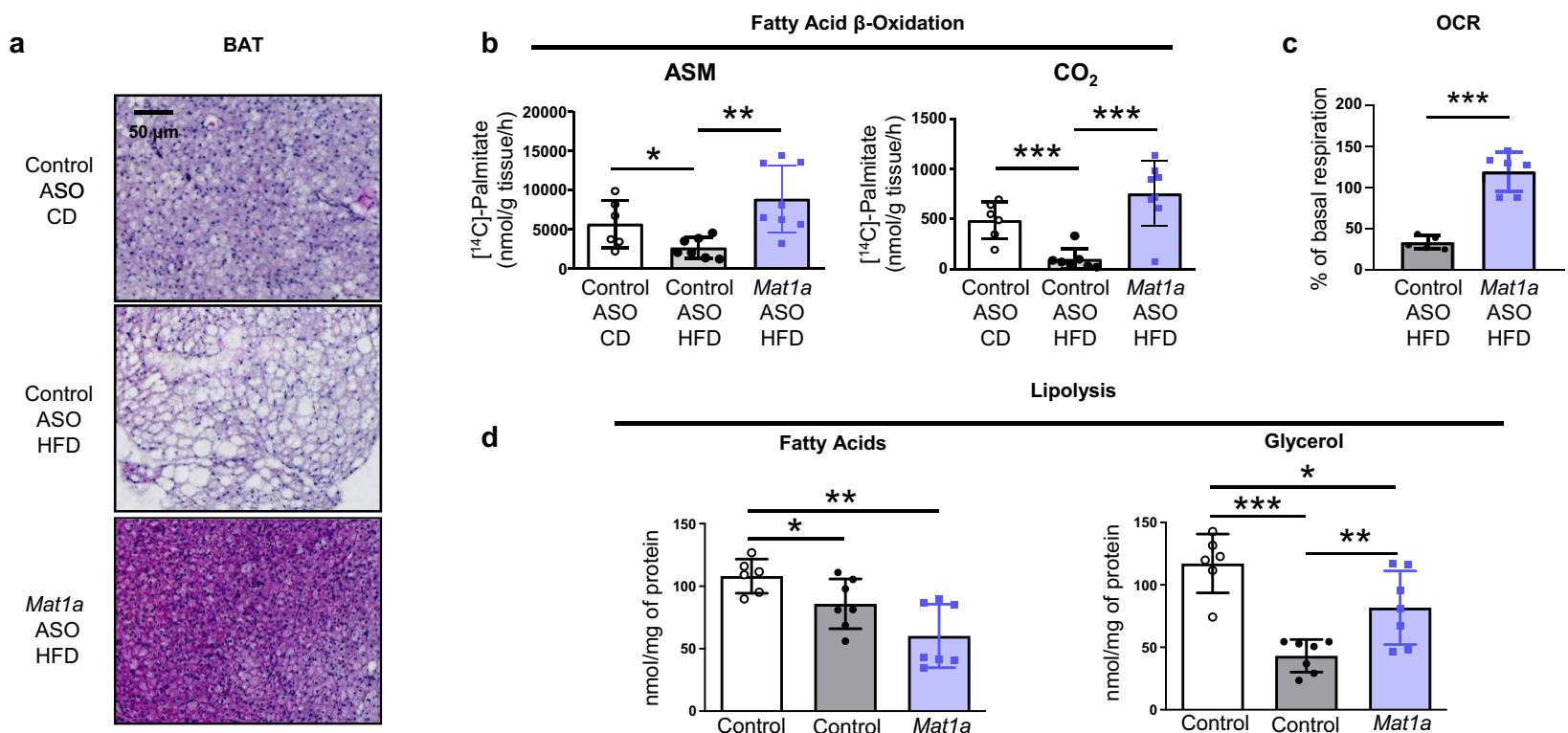

d

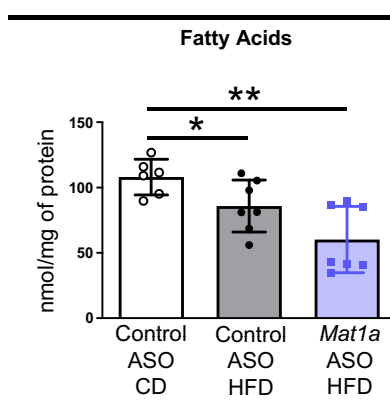

Lipolysis

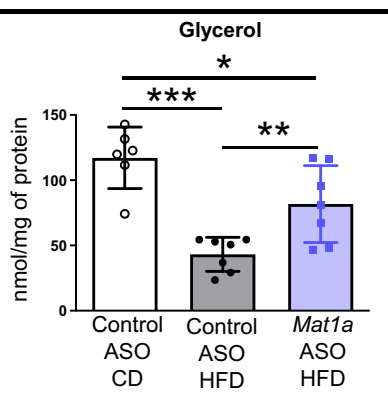

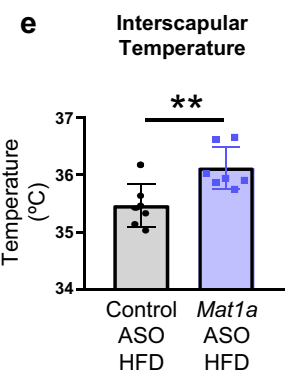

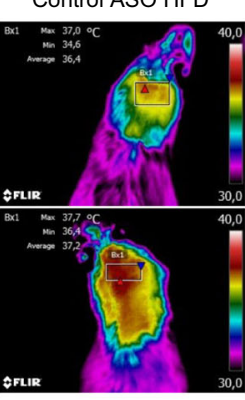

Mat1a ASO HFD

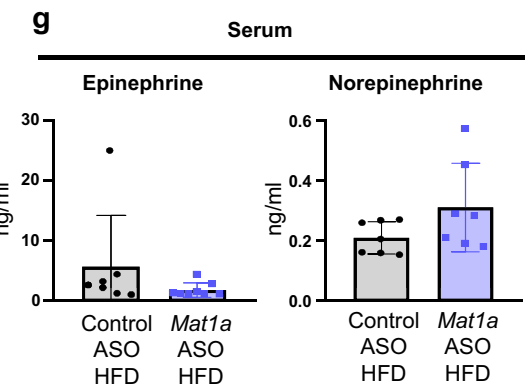

f
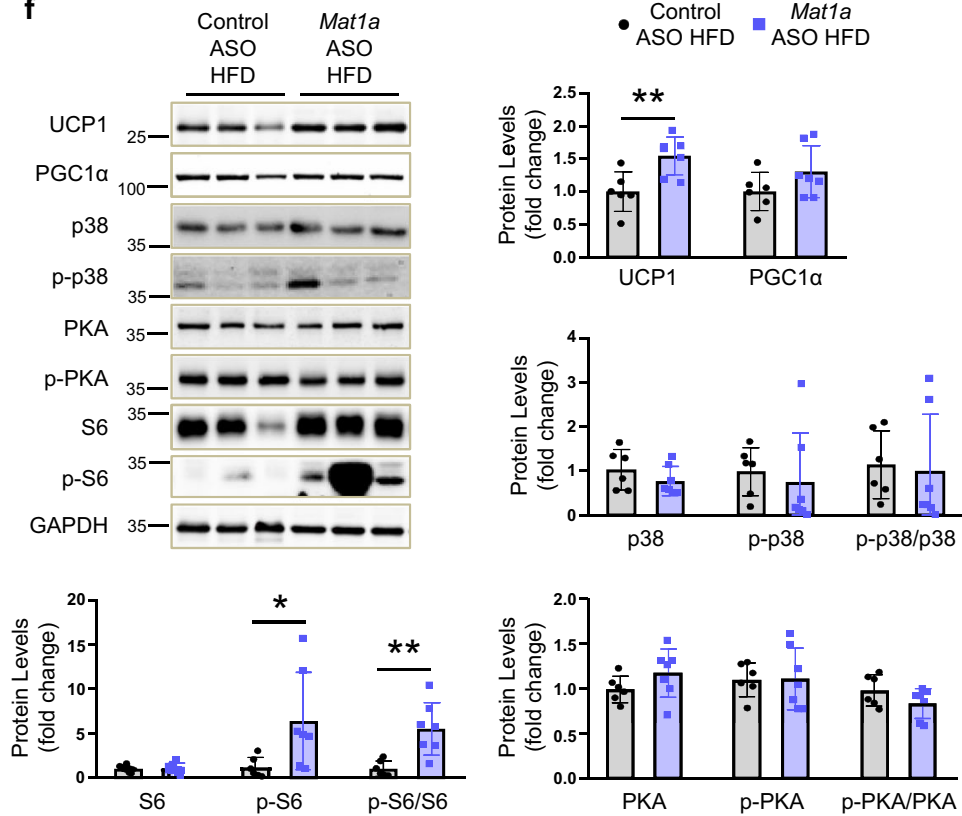

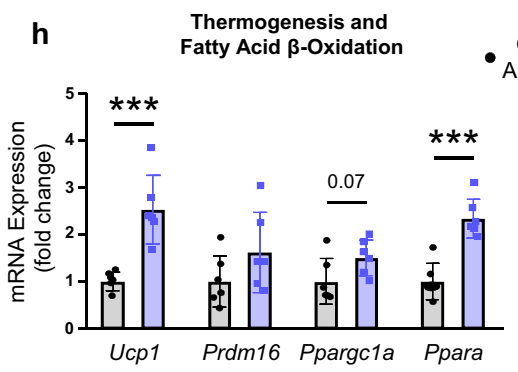

Control Mat1a
ASO HFD
ASO HFD

Adipose Tissue
Differentation

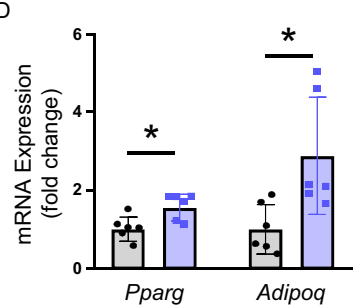

Mat1a ASO from the sixth week (one IP dose per week) (Fig. 3a). Furthermore, although dispersion of the data was high, the Sirius Red and F4/80 immunostaining (Fig. 3a) and the expression levels of genes involved in fibrosis and inflammation (Supplementary Fig. 6a), showed that treatment with Mat1a ASO did not induce the increase of liver fibrosis or inflammation. To validate the antisteatotic effect of Mat1a knockdown, another cohort of HFD-fed mice were treated with Mat1a ASO2, and Mat1a-KO mice were fed a HFD. Similar results were obtained: both Mat1a ASO2 treated (Supplementary Fig. 6b, c) and Mat1a-KO (Supplementary Fig. 6d) mice did not develop hepatosteatosis as evident from the absence of liver lipid droplets and reduced 
Fig. 2 Mat1a antisense oligonucleotides induce thermogenesis in brown adipose tissue (BAT). Two-month-old C57BL/6J mice were fed a chow diet (CD) or a high-fat diet (HFD) for 10 weeks. During the last 4 weeks mice were treated with Mat1a antisense oligonucleotide (ASO) or control ASO (25 mg/ $\mathrm{kg} /$ week) until sacrifice. a Representative microphotographs of BAT sections stained with Hematoxylin/Eosin of CD- $(n=6)$ and HFD-fed ASO-treated mice $(n=6)$. $\mathbf{b}$ Fatty acid $\beta$-oxidation was determined measuring the amount of $\left[{ }^{14} \mathrm{C}\right]$-acid-soluble metabolites (ASM) (incomplete oxidation of palmitate) and $\left[{ }^{14} \mathrm{C}\right]-\mathrm{CO}_{2}$ (complete oxidation of palmitate) in BAT of CD- $(n=6)$ and HFD-fed control $(n=7)$ and Mat7a $(n=8)$ ASO-treated mice. c Oxygen consumption rate (OCR) expressed as $\%$ of basal respiration of HFD-fed control $(n=5)$ and Mat7a $(n=6)$ ASO-treated mice. d BAT lipolysis was determined by measuring the amount of fatty acids and glycerol secreted ex vivo by BAT of CD- $(n=6)$ and HFD-fed control $(n=7)$ and Mat7a $(n=7)$ ASO-treated mice. e Quantification and infrared thermal images of BAT interscapular temperature of HFD-fed control $(n=7)$ and Mat1a $(n=7)$ ASOtreated mice. $\mathbf{f}$ Representative blots and densitometries of uncoupling protein1 (UCP1), peroxisome proliferator- $\gamma$ activated receptor (PPAR)- $\gamma$ co-activator $1 \alpha(P G C 1 \alpha)$, phosphorylated and total forms of mitogen-activated protein kinase p38 (p38), protein kinase A (PKA) and S6 protein, and glyceraldehyde-3phosphate dehydrogenase (GAPDH), as representative loading control, in BAT of HFD-fed control $(n=6)$ and Mat7a $(n=7)$ ASO-treated mice. The ratio between phosphorylated and total forms of the proteins was determined. $\mathbf{g}$ Serum epinephrine and norepinephrine levels in HFD-fed control $(n=7)$ and Mat1a $(n=7)$ ASO-treated mice. $\mathbf{h}$ mRNA expression levels in BAT of HFD-fed control $(n=6)$ and Mat1a $(n=6)$ ASO-treated mice of Ucp1, PR/SET Domain 16 (Prdm16), PGC1 $\alpha$ (Ppargc1a) and PPAR alpha (Ppara), as indicators of thermogenesis and mitochondrial fatty acid $\beta$-oxidation), and PPAR gamma (Pparg) and adiponectin (Adipoq) genes, as indicators of adipocyte differentiation. Results were normalized with Gapdh and Actin (Actb). Values are presented as means \pm SD. Statistically significant differences between groups are indicated by ${ }^{\star} p<0.05,{ }^{\star \star} p<0.01$, and ${ }^{\star \star *} p<0.001$ (two-tailed Student's test). Source data are provided as a Source data file.

TG levels (Supplementary Fig. 6b, d). In addition, targeting liver Mat1a also induced a decrease in muscle TG levels (Supplementary Fig. 7a).

One of the most important metabolic processes regulating liver lipid content is the mitochondrial FAO. Our results showed that targeting Mat1a in HFD-fed mice did not induce changes in FAO when compared with controls, as demonstrated by levels of $\left[{ }^{14} \mathrm{C}\right]-$ Palmitate oxidation and serum ketone bodies concentration (Fig. 3b). In contrast, de novo lipogenesis fluxes, assessed in liver pieces, showed a marked decrease (Fig. 3c), mainly related to the reduction in levels of ACC and FAS proteins, key enzymes involved in de novo fatty acid synthesis (Fig. 3d). Thus, the results here demonstrated that targeting Mat1a improves the obesityrelated hepatosteatosis (Fig. 3).

We next analyzed if this antisteatotic profile was also linked to reduced levels of serum TG. The results showed that during feeding and fasting, Mat1a ASO treatment reduced serum TG levels when compared to control ASO-treated mice (Fig. 4a). In vivo analysis of TG-rich lipoprotein metabolism showed that hepatic TG secretion rate maintained unaltered in HFD-fed Mat1a ASO-treated mice when compared to the control ASOtreated mice (Fig. 4b) whereas the chylomicron TG clearance was increased (Fig. 4c). To ascertain the fate of the dietary lipids when Mat $1 a$ was knocked-down in vivo, a bolus of olive oil with $\left[{ }^{3} \mathrm{H}\right]-$ Triolein was administrated. The results showed that when HFDfed mice were treated with Mat1a ASO, the uptake of dietary lipids was mainly increased in the BAT (Fig. 4d). This increase was not linked with higher BAT levels of lipoprotein lipase (LPL), involved in TG-rich lipoprotein catabolism, or of CD36, a fatty acid transporter (Fig. 4e). However, serum levels (Fig. 4f) and liver expression (Fig. $4 \mathrm{~g}$ ) of the LPL activator ApoC2, were increased, while those of the LPL inhibitor, ApoC3 were decreased (Fig. 4f, g). These results suggest that when targeting liver Mat1a, serum TGs are transported towards the BAT, which will also contribute to a decreased accumulation in liver.

Mat1a ASO reduces DIO by increasing energy expenditure and increases BAT TG uptake, suggesting a higher utilization of lipids in BAT to cope with energy requirements for its thermogenic action, which could also contribute to the decreased hepatic lipid content in these mice.

Mat1a ASOs reverse obesity and hepatosteatosis in ob/ob mice. To demonstrate the improvement in obesity, insulin resistance and hepatosteatosis induced by Mat1a ASO in genetically obese mice, leptin deficient $o b / o b$ mice, a well-established model of obesity 22 were used. Changes in body weight were assessed during the ASO treatment; targeting hepatic Mat1a prevented the weight gain after the second dose and induced weight loss by the end of the treatment (Fig. 5a). As previously observed in DIO mice, the food intake maintained unchanged (Fig. 5b). Notably, Mat1a ASO improved GTT (Fig. 5c), ITT (Fig. 5d), fasting insulin levels (Fig. 5e) and induced a marked resolution of hepatosteatosis with a reduced accumulation of lipid droplets and liver TG concentration (Fig. 5f). The results further showed that targeting Mat1a ASO in $o b / o b$ mice led to increased FAO in BAT (Fig. 5g), increased protein levels and expression of UCP1 in BAT (Fig. 5h, i) and increased expression of Ppara, Pparg and Adipoq in BAT (Fig. 5i); thus, confirming the role of Mat1a-induced BAT thermogenesis in the improved phenotype.

Mat1a ASOs prevent obesity through induction of hepatocyte secretion of FGF21. Mat1a ASO prevents and reverses obesity by inducing BAT thermogenesis. The products of Mat1a gene (MATI/III) catalyze the first reaction of the methionine cycle in liver ${ }^{6}$, which is the conversion of methionine into SAMe. Thus, the loss of Mat1a results in the reduction of methionine utilization and a concomitant accumulation of liver methionine and the decrease in SAMe levels ${ }^{6}$. In concordance, the results here showed that liver methionine was increased $610 \%$ in Mat1a ASO-treated HFD-fed mice liver and 2350\% in HFD-fed Mat1a-KO mice liver when compared to the corresponding WT mice (Supplementary Fig. $8 \mathrm{a}, \mathrm{b})$. This increase was linked with a decrease in liver SAMe of $20 \%$ in Mat $1 a$ ASO-treated mice and of $30 \%$ in Mat1a-KO mice when compared to the corresponding controls (Supplementary Fig. 8a, b). Considering that methionine deficiency induces thermogenesis through the secretion of liver FGF2 $1^{23}$, to asses if the decreased utilization led also to the secretion of FGF21, levels of FGF21 were also measured in serum of the generated models. Serum levels of FGF21 in HFD-fed Mat1a ASO and Mat1a ASO2 treated mice, in Mat1a ASO-treated ob/ob and Mat1a-KO mice fed a HFD were increased (Fig. 6a). In vitro experiments showed that hepatocytes isolated from Mat1a ASOtreated HFD-fed mice secreted more FGF21 into the media than hepatocytes isolated from control ASO-treated mice (Fig. 6b). To investigate if hepatocyte FGF21 was directly involved in the improvement of obesity, AlbCre-Fgf21 and their control mice (AlbCre) were fed a HFD and were treated with Mat1a or control ASO from the sixth week of the HFD. Targeting Mat1a in HFDfed AlbCre mice resulted in body weight loss (Fig. 6c and Supplementary Fig. 8c) without inducing changes in food intake (Supplementary Fig. 8d) whereas it did not affect body weight or food intake when Fgf21 was absent in the liver (Fig. 6c and 
a

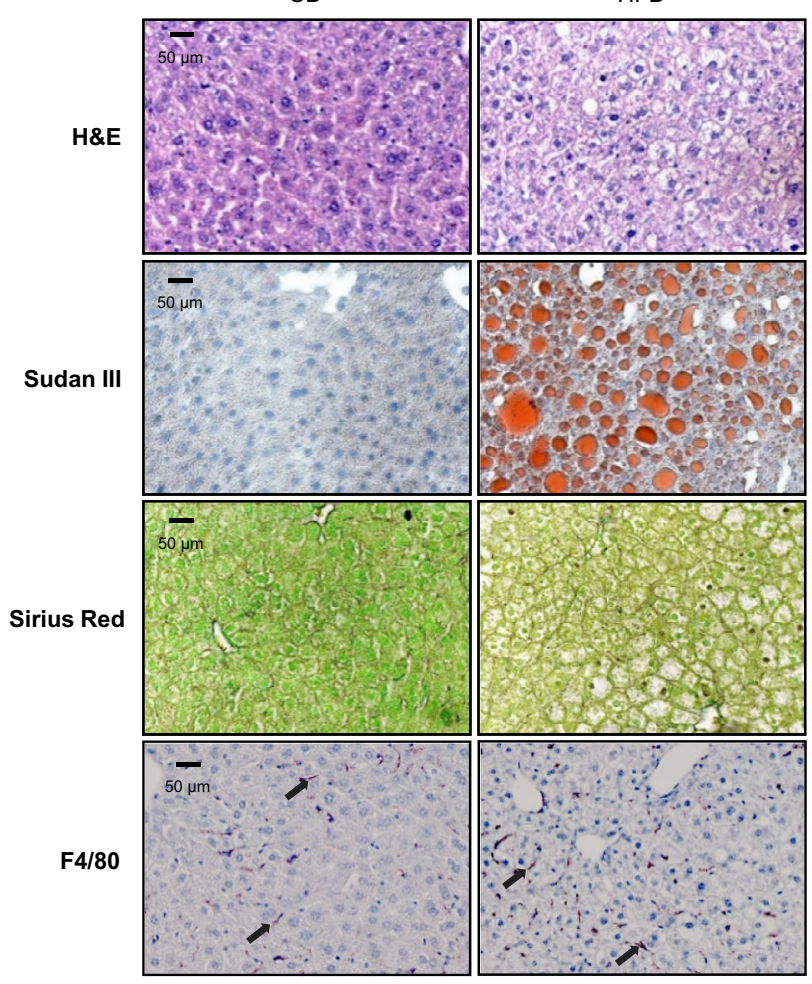

b

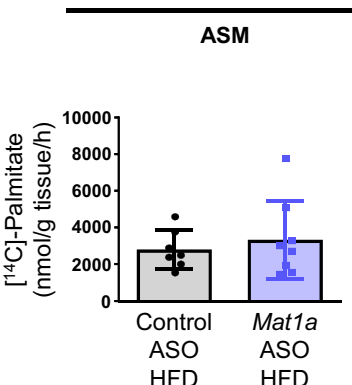

Fatty Acid $\beta$-Oxidation

$\mathrm{CO}_{2}$

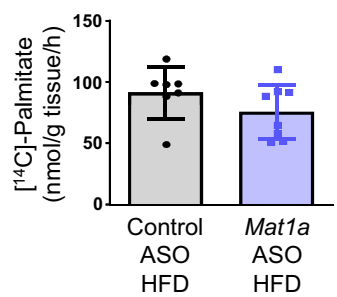

Ketone Bodies

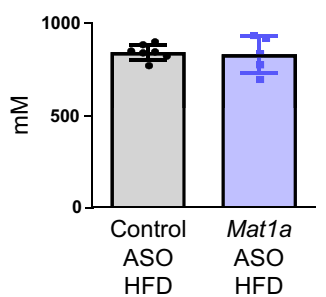

Liver Triglyceride
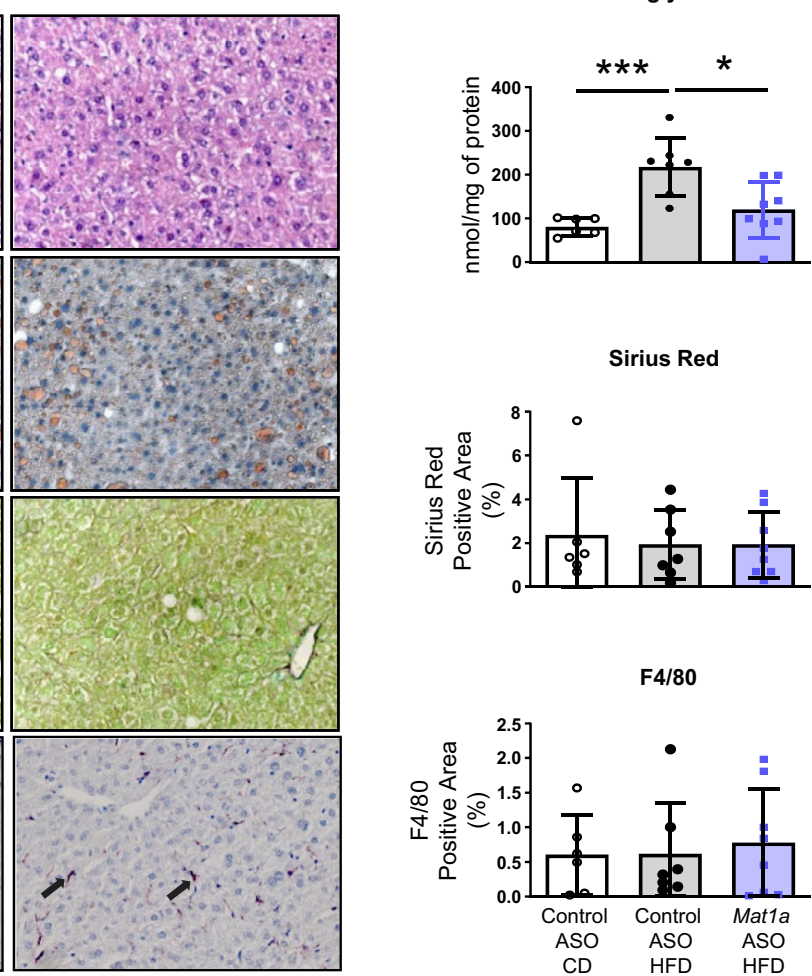

$\mathrm{F} 4 / 80$

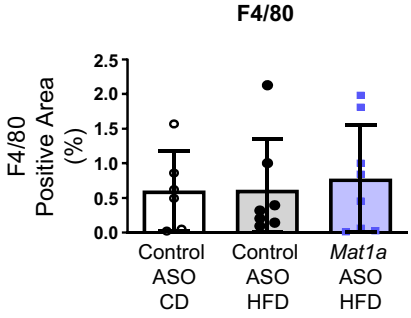

c

de novo Lipogenesis of TG

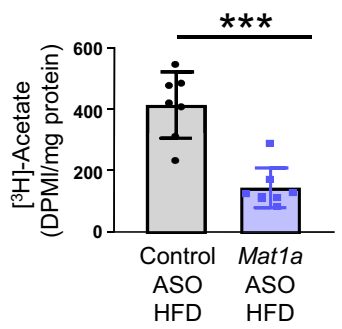

d

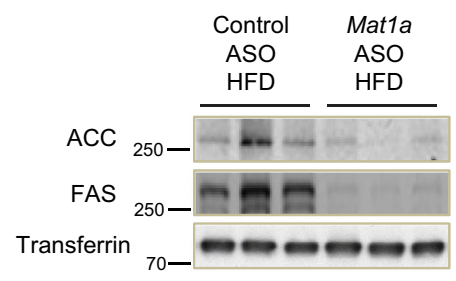

$\begin{array}{cc}\text { Control } & \text { Mat1a } \\ \text { ASO HFD } & \text { ASO HFD }\end{array}$

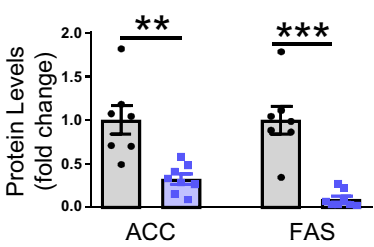

Supplementary Fig. 8d). As expected, Mat1a ASO treatment increased FGF21 in serum of HFD-fed AlbCre mice while it did not affect FGF21 levels in HFD-fed AlbCre-Fgf21 mice, in which serum FGF21 levels were hardly detectable (Fig. 6d).

The increased energy expenditure (Fig. 6e), and BAT complete FAO (Fig. 6f) in Mat1a ASO-treated HFD-fed AlbCre mice was not observed when Fgf21 was absent in hepatocytes (Fig. 6e, f). The results also showed that the increased UCP1 levels and S6 signaling (Fig. 6g) induced by Mat1a ASO was not found when Fgf21 was knocked out (Fig. 6g). Targeting Mat1a did not affect serum epinephrine nor norepinephrine levels in hepatocyte-specific Fgf21-KO mice (Supplementary Fig. 8e). However, the altered ApoCs expression observed in the liver when targeting Mat1a, was no longer observed when $F g f 21$ was absent (Supplementary Fig. 8f).

To ascertain if the receptor of FGF21, the $\beta$-klotho, and UCP1 were involved in the increased thermogenesis, Ucp 1 and $\beta$-Klotho were silenced in BAT (Supplementary Fig. 9a). 
Fig. 3 Mat1a antisense oligonucleotides protect from high-fat diet (HFD)-induced hepatosteatosis. Two-month-old C57BL/6 J mice were fed a chow $\operatorname{diet}(\mathrm{CD})$ or a high-fat diet (HFD) for 10 weeks. During the last 4 weeks mice were treated with Mat1a antisense oligonucleotide (ASO) or with control ASO (25 mg/kg/week) until sacrifice. a Representative microphotographs of liver sections stained with Hematoxylin/Eosin (H\&E), Sudan III, Sirius Red and F4/ 80 , and liver triglyceride (TG) content, and quantification of Sirius red and F4/80 in CD- $(n=6)$ and HFD-fed control $(n=7)$ and Mat7a $(n=8)$ ASOtreated mice. $\mathbf{b}$ Liver fatty acid $\beta$-oxidation was determined measuring the amount of $\left[{ }^{14} \mathrm{C}\right]$-acid-soluble metabolites (ASM) (incomplete oxidation of palmitate), $\left[{ }^{14} \mathrm{C}\right]-\mathrm{CO}_{2}$ (complete oxidation of palmitate) and serum ketone bodies levels in HFD-fed control $(n=7$ for ketone bodies and $n=7$ for $\beta$-oxidation) and Mat7a ( $n=5$ for ketone bodies and $n=8$ for $\beta$-oxidation) ASO-treated mice. $\mathbf{c}$ Liver TG de novo lipogenesis determined by incorporation of $\left[{ }^{3} \mathrm{H}\right]$-acetate into TG in HFD-fed control $(n=7)$ and Mat1a $(n=8)$ ASO-treated mice liver pieces. $\mathbf{d}$ Representative blots and densitometries of acetylcoenzyme A carboxylase (ACC), fatty acid synthase (FAS) and transferrin, as representative loading control, in liver of HFD-fed control ( $n=7)$ and Mat7a $(n=8)$ ASO-treated mice. Values are presented as means \pm SD. Statistically significant differences between groups are indicated by ${ }^{\star} p<0.05,{ }^{\star \star} p<0.01$, and ${ }^{\star \star *} p<0.001$ (two-tailed Student's test). Source data are provided as a Source data file.

The results showed that the body weight loss (Supplementary Fig. 9b, c) and the increased energy expenditure (Supplementary Fig. 9d) induced by Mat1a ASO, were not evident when Ucp1 or $\beta$-Klotho were silenced in BAT. To note, all these effects were independent of changes in locomotor activity (Supplementary Fig. 9e).

Finally, compared with control ASO treatment, HFD-fed AlbCre mice also exhibited improved GTT when given Mat1a ASO (Fig. 6h); HFD-fed AlbCre-Fgf21mice on the other hand did not show differences in GTT between ASO treatments (Fig. 6h).

These data collectively show that targeting Mat1a activates the liver-BAT axis through the induction of hepatocyte FGF21 secretion.

NRF2 mediates hepatocyte FGF21 secretion. The most studied regulator of FGF21 has been the PPARa ${ }^{24}$; however, as chronic deficiency in Mat1a leads to endoplasmic reticulum (ER) stress ${ }^{19}$ and ER stress may also regulate generation of FGF21 through activation of activating transcription factor 4 (ATF4) ${ }^{25}$, we hypothesized that this might be an important regulator of FGF21 in this context. In addition, the transcription factor NRF2, an ATF4interacting factor, is a master regulator of the cellular defense system against oxidative stress and has been shown to modulate FGF21 expression in diabetic mice ${ }^{26}$. Thus, here PPAR $\alpha$, ATF4 and NRF2 were evaluated as potential regulators of FGF21 in our models. Experiments performed in hepatocytes isolated from HFDfed mice treated with ASOs, in which Nrf2, Atf4 or Ppara were silenced through the usage of siRNAs (Supplementary Fig. 10a) showed that the knockdown of Nrf2 was able to reduce the increased FGF21 secretion induced by Mat1a ASO in HFD-fed mice hepatocytes (Supplementary Fig. 10b). However, Atf4 or Ppara knockdown did not reduce FGF21 secretion (Supplementary Fig. 10b). Targeting Mat1a did not induce changes in the levels of nuclear PPARa or ATF4 (Supplementary Fig. 10c) and when hepatocytes were treated with inhibitors of PPAR $\alpha$ (Supplementary Fig. 10d) or of ER stress (Supplementary Fig. 10e), neither was able to recover normal levels of FGF21 secretion (Supplementary Fig. 10d, e).

Here, we found that when isolated hepatocytes from ASOtreated HFD-fed mice were exposed to ML385, a specific inhibitor of NRF2 activity, FGF21 secretion decreased (Fig. 7a), in concordance with the decreased secretion induced by siNrf2 (Supplementary Fig. 10b). Moreover, when HFD-fed mice were treated with Mat1a ASO, liver NRF2 levels increased in nucleus (Fig. 7b), while levels in liver remained unaltered and those in liver cytoplasm decreased (Fig. 7b), and NRF2-target genes were upregulated (Fig. 7c), thus confirming that targeting Mat1a increases NRF2 activity. Even more, in hepatocytes from HFDfed mice treated with Mat1a ASO, NRF2 was found directly binded to a promoter site in the Fgf21 gene (Fig. 7d). These results indicate that NRF2 plays a key role in mediating Mat1a ASO-induced hepatocyte FGF21 secretion. Next, we investigated if the protection from hepatosteatosis induced by targeting Mat1a was directly mediated by FGF21 or NRF2. The results showed that in the HFD-fed AlbCre-Fgf21 mice (where Fgf21 was absent in hepatocytes), NRF2 levels were preserved increased in nucleus (Fig. 7e), confirming that NRF2 is upstream FGF21. However, concentrations of liver TGs were enhanced when compared with HFD mice treated with the control ASO (Fig. 7f). These data imply that while Mat1a ASO increases NRF2 and this transcription factor is upstream FGF21, the deletion of FGF21 is sufficient to prevent the actions of Matla ASO treatment. In addition, the results showed that targeting liver Mat1a also induced the expression of Nrf2 and, more markedly, Fgf21 in BAT (Supplementary Fig. 11a), and that this increased expression was absent when liver Fgf21 was absent (Supplementary Fig. 11b) or when BAT primary cells were maintained in culture (Supplementary Fig. 11c, d). Thus, the Mat1a ASO induced secretion of FGF21 by hepatocytes also regulates the expression of Fgf 21 in BAT.

Mat1a-KO hepatocytes, with low hepatic SAMe levels, have higher mitochondrial reactive oxygen species (ROS), which are normalized when Mat1a is overexpressed ${ }^{27}$. SAMe is required in the transsulfuration pathway (Fig. $7 \mathrm{~g}$ ). In this sense, we found that levels of glutathione (GSH) were decreased in Mat1a ASOtreated HFD-fed mice as compared to the ASO control treated mice while glutathione disulfide (GSSG) levels maintained unaltered (Fig. 7g) and levels of malondialdehyde (MDA), a measure of lipid peroxidation, were increased (Fig. $7 \mathrm{~g}$ ). Treatment of hepatocytes with GSH ether or the antioxidant $\mathrm{N}$-acetylcysteine (NAC), decreased the Mat1a ASO-induced secretion of FGF21 (Fig. 7h), the same effect was obtained when hepatocytes were treated with SAMe (Fig. 7i), which effects inside the hepatocytes were verified by the increased expression of genes involved in the methionine cycle (Supplementary Fig. 12a) and the levels of small ubiquitin-related modifier 1 (SUMO1)conjugated proteins and SUMO1 (Supplementary Fig. 12b). Thus, the results show that SAMe deficiency is involved in the increased FGF21 secretion by hepatocytes.

Given that methionine accumulates in hepatocytes due to Mat1a ASO treatments, we further incubated hepatocytes in a methionine-deficient media. The results showed that whilst the methionine-deficient media increased FGF21 secretion in control ASO-treated hepatocytes (Supplementary Fig. 12c); it did not affect secretion of FGF21 in Mat1a ASO-treated cells (Supplementary Fig. 12c).

Altogether, these data show that treatment with Mat1a ASO increases ROS despite regression of hepatosteatosis (and without fibrosis or inflammation).

\section{Discussion}

Obesity is a major public health issue with increasing prevalence worldwide. It is a well-established risk factor for a variety of diseases, including NAFLD $^{28}$, type 2 diabetes $^{29}$, dyslipemia ${ }^{30}$, among others. Weight loss through pharmacological treatments, 

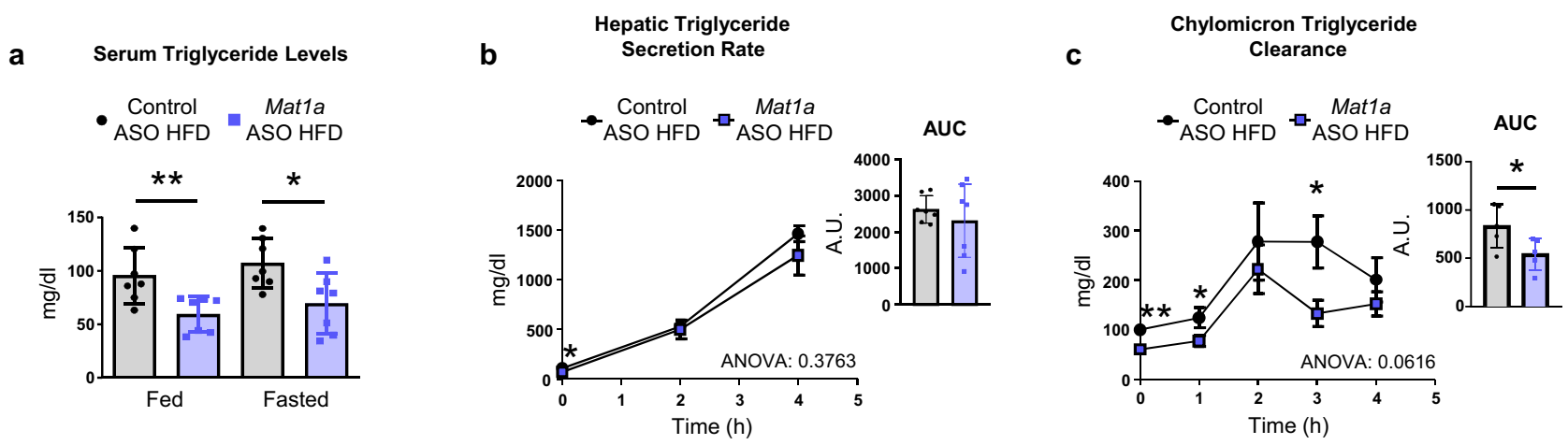

d

BAT
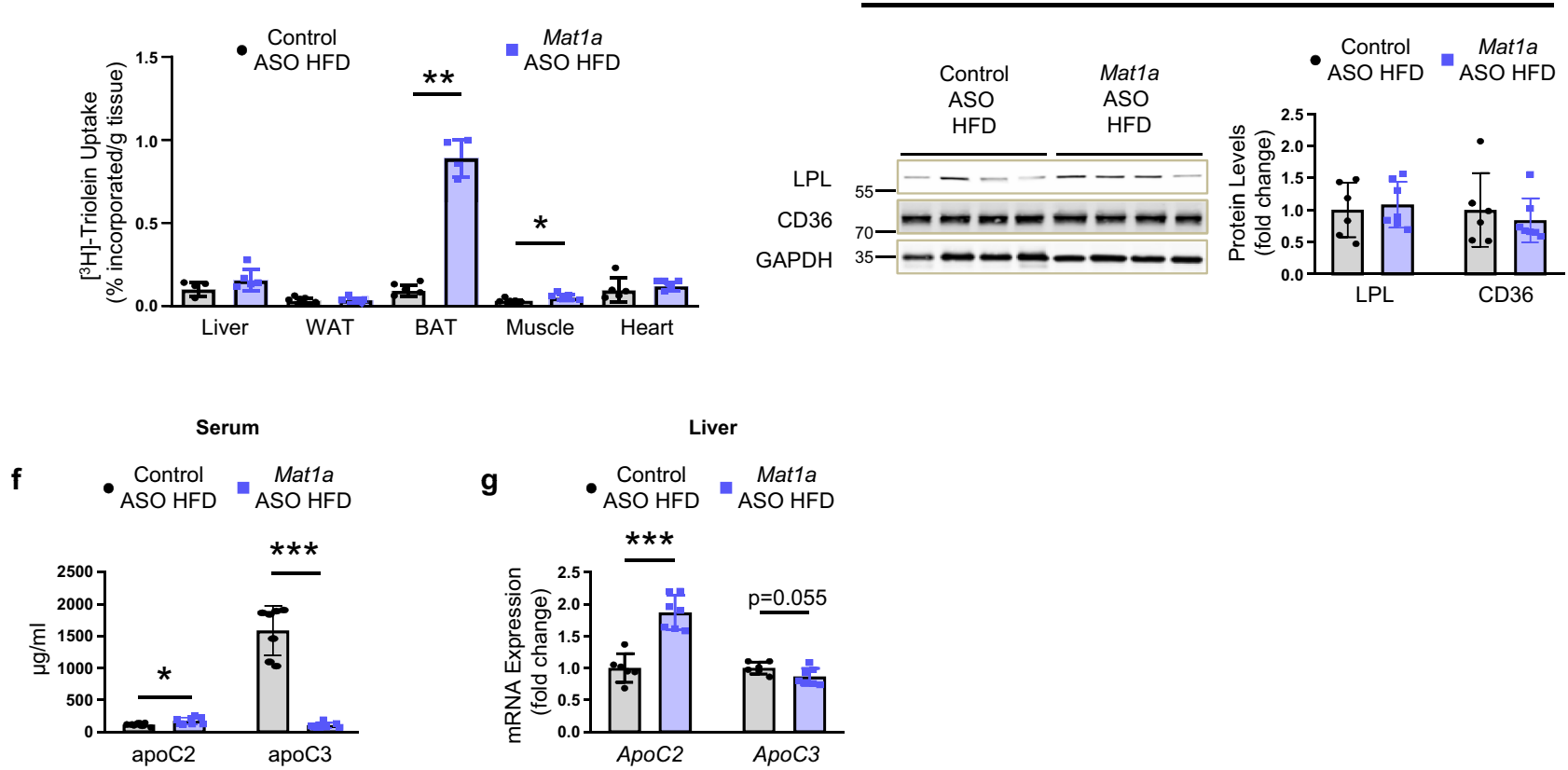

Fig. 4 Mat1a antisense oligonucleotides channel plasma lipids towards the brown adipose tissue (BAT). Two-month-old C57BL/6J mice were fed a high-fat diet (HFD) for 10 weeks. During the last 4 weeks mice were treated with Mat1a antisense oligonucleotide (ASO) or with control ASO (25 mg/kg/ week) until sacrifice. a Serum triglycerides (TG) levels in fed and fasted conditions in HFD-fed control $(n=7)$ and Mat7a $(n=7)$ ASO-treated mice. b Circulating TG levels from mice fasted for $4 \mathrm{~h}$ prior $(\mathrm{O} \mathrm{h}), 2 \mathrm{~h}$ and $4 \mathrm{~h}$ after treatment with the LPL inhibitor, poloxamer P-407 in HFD-fed control ( $n=7$ ) and Mat1a $(n=7)$ ASO-treated mice. Data are also indicated as area under the curve (AUC) expressed in arbitrary units (A.U.). c TG serum levels during oral lipid tolerance test after overnight fasting in HFD-fed control $(n=5)$ and Mat7a $(n=5)$ ASO-treated mice. Data are also indicated as AUC expressed in A.U. $\mathbf{d}$ Tissue distribution of $\left[{ }^{3} \mathrm{H}\right]$-triolein $4 \mathrm{~h}$ after oral gavage in HFD-fed control $(n=5)$ and Mat7a $(n=5)$ ASO-treated mice. e Representative blots and densitometries of lipoprotein lipase (LPL), fatty acid translocase (CD36) and glyceraldehyde-3-phosphate dehydrogenase (GAPDH) in BAT of HFD-fed control $(n=6)$ and Mat1a $(n=7)$ ASO-treated mice. $\mathbf{f}$ Serum apoC2 and apoC3 levels in HFD-fed control $(n=7)$ and Mat1a $(n=8)$ ASO-treated mice. g mRNA liver expression of ApoC2 and ApoC3 levels in HFD-fed control $(n=6)$ and Mat7a $(n=7)$ ASO-treated mice. Results were normalized with Gapdh. Values are presented as means \pm SEM for time course representations, and as means \pm SD for histograms. Statistically significant differences between groups are indicated by ${ }^{\star} p<0,05,{ }^{* \star} p<0.01$, and ${ }^{* * *} p<0.001$ (two-tailed Student's test). Statistical analysis performed by two-way ANOVA test comparing Control ASO HFD vs. Mat1a ASO HFD is presented in hepatic TG secretion and chylomicron clearance curves. Source data are provided as a Source data file.

lifestyle interventions and/or bariatric surgery are also effective to treat the obesity-related co-morbidities ${ }^{3}$. However, there is still a need to better understand the connecting relationships between different metabolic disorders related to obesity so that new therapies might be proposed.

We demonstrate that ASO-mediated silencing of Mat1a reverses and prevents obesity, insulin resistance and the associated hepatosteatosis as well as reducing lipids in serum. Mat1a gene is mainly expressed in the liver (primarily in hepatocytes) and encodes the methionine adenosyltransferase subunit alpha 1 (MATa1). The oligomerization of MATa1 leads to the formation of the enzyme MATI/III, which is the first enzyme of the methionine cycle ${ }^{6,18}$. MATI/III catalyzes the conversion of methionine to SAMe, a major methyl donor in the cell ${ }^{18}$. Altered methionine metabolism and SAMe synthesis have been associated with adiposity and weight gain in individuals with overweight and obesity ${ }^{31}$.

These results showing that inhibition of Mat1a alleviates obesity and obesity-associated metabolic diseases are consistent with previous reports, which showed that disruption of other enzymes such as PEMT $^{12}$ or NNMT ${ }^{15}$ involved in the methionine cycle leads to resistance to obesity. Some metabolic beneficial 
a

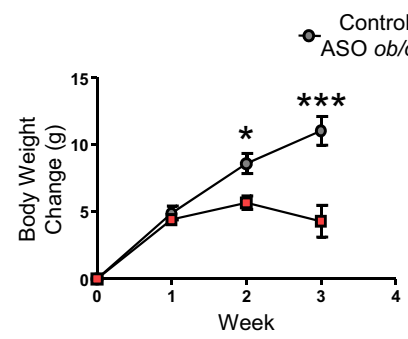

C
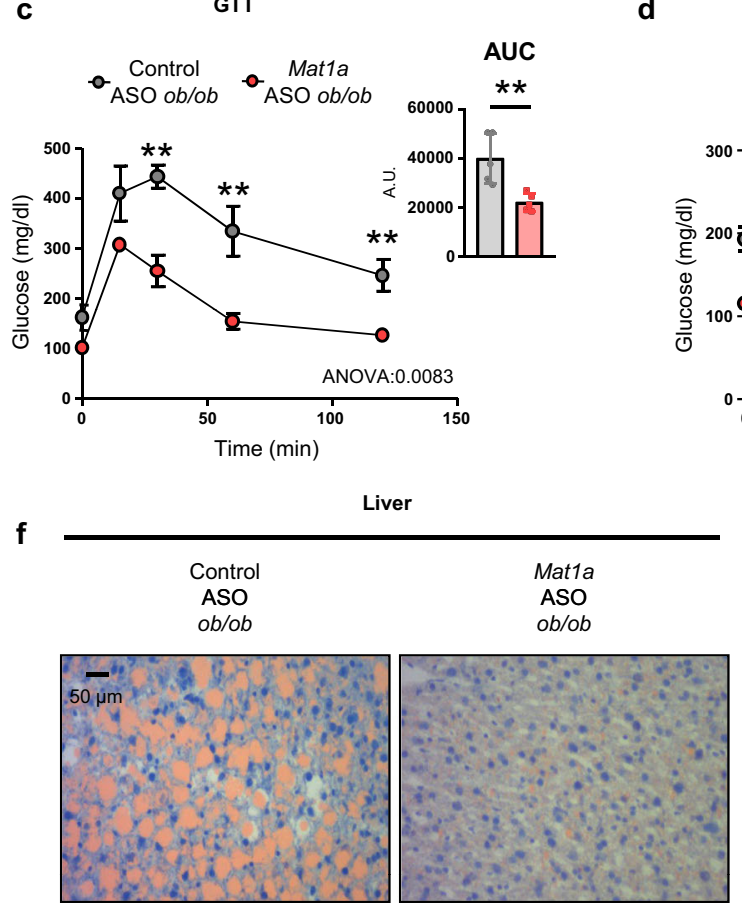

Triglyceride
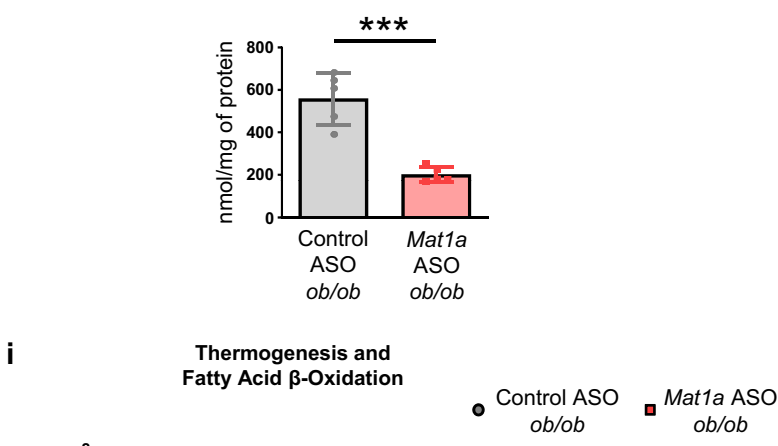

d
ASO ob/ob

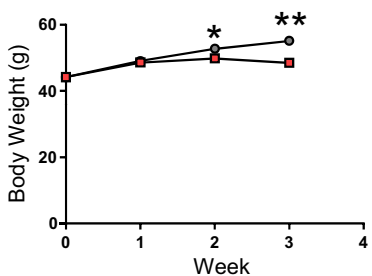

b

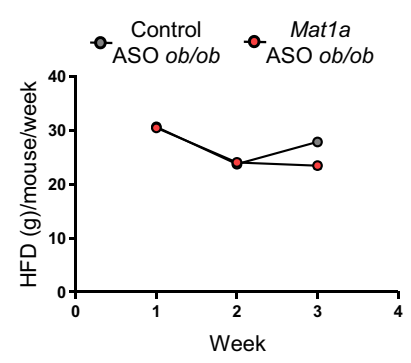

Food Intake

e Insulin
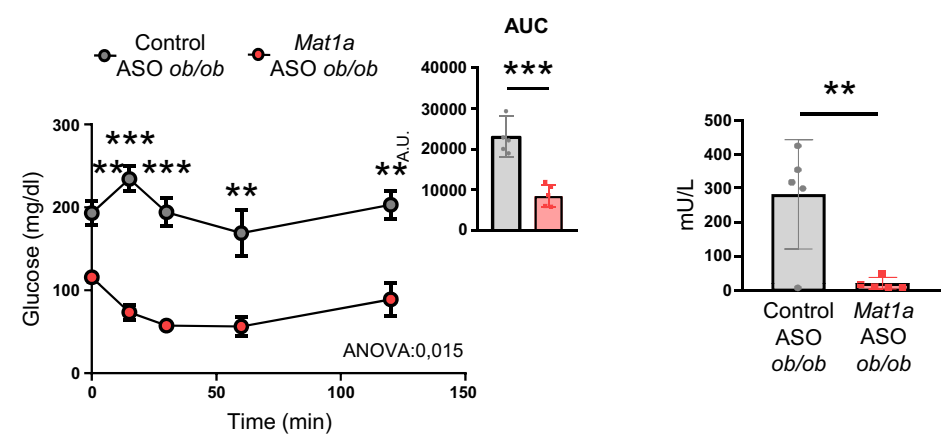

Fatty Acid $\beta$-Oxidation in BAT

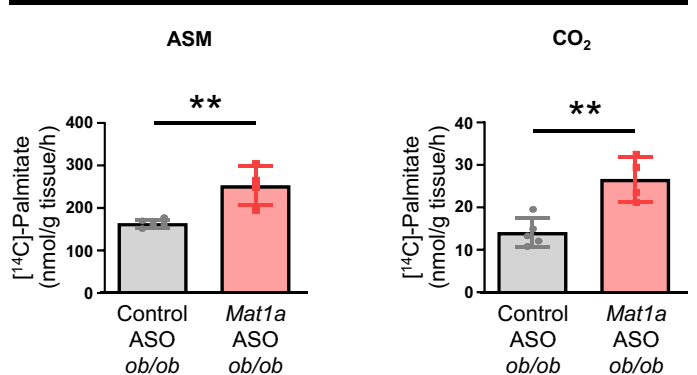

h

BAT

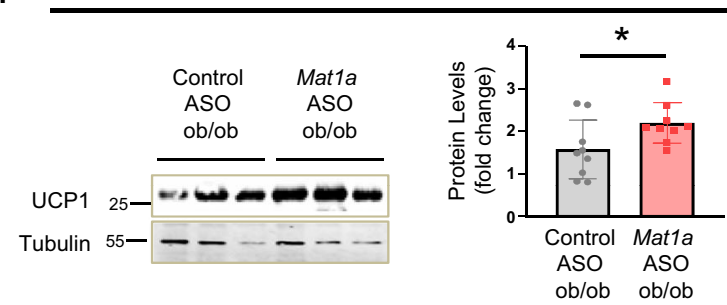

Adipose Tissue

Differentiation

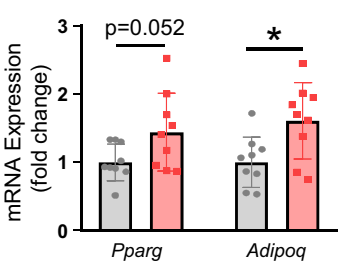

effects were also obtained in the absence of enzymes such as cystathionine- $\beta$-synthase or cystathionine gamma-lyase ${ }^{31}$, involved in the transsulfuration pathway, which is strongly supported by the flux of methionine.

In the context of obesity, the beneficial effects driven by Mat 1a deficiency closely resembles those produced by dietary MR. In

MR diets, SAMe synthesis is altered as when there is a downregulation in Mat1a. MR leads to weight loss, improvement of insulin resistance and adiposity ${ }^{32,33}$, reduction of hepatosteatosis and dyslipemia ${ }^{33}$, and browning of adipose tissue activating its FAO and lipolysis ${ }^{34}$. The main mechanism by which MR induces its positive effects is the hepatic secretion of FGF $21^{23}$. Our results 
Fig. 5 Mat1a antisense oligonucleotides reverse obesity and hepatoesteatosis in ob/ob mice. Three-month-old B6.Cg-Lepob/J (ob/ob) mice were fed a high-fat diet (HFD) for 4 weeks. During the diet mice were treated with Mat1a antisense oligonucleotide (ASO) or control ASO (25 mg/kg/week) until sacrifice. a Body weight change and body weight for HFD-fed control $(n=5)$ and Mat7a $(n=5)$ ASO-treated ob/ob mice. b Food intake for HFD-fed control ( $n=2$ cages) and Mat1a ( $n=2$ cages) ASO-treated ob/ob mice. c Glucose (GTT) and $\mathbf{d}$ insulin tolerance tests (ITT) in HFD-fed control ( $n=5)$ and Mat1a $(n=5)$ ASO-treated ob/ob mice. Data are also indicated as area under the curve (AUC) expressed in arbitrary units (A.U.). e Serum insulin levels in HFDfed control $(n=5)$ and Mat1a $(n=5)$ ASO-treated ob/ob mice fasted overnight. $\mathbf{f}$ Representative microphotographs of liver sections stained for Sudan III and liver triglyceride (TG) concentration of HFD-fed control $(n=5)$ and Mat1a $(n=5)$ ASO-treated ob/ob mice. $\mathbf{g}$ BAT fatty acid $\beta$-oxidation, determined measuring the amount of $\left[{ }^{14} \mathrm{C}\right]$-acid-soluble metabolites (ASM) (incomplete oxidation of palmitate) and $\left[{ }^{14} \mathrm{C}\right]-\mathrm{CO}_{2}$ (complete oxidation of palmitate) in HFD-fed control $(n=5)$ and Mat1a $(n=4)$ ASO-treated ob/ob mice. $\mathbf{h}$ Representative blots and densitometries of uncoupling protein1 (UCP1) and tubulin in BAT of HFD-fed control $(n=9)$ and Mat1a $(n=9)$ ASO-treated ob/ob mice. i mRNA expression levels in BAT of HFD-fed control $(n=9)$ and Mat1a $(n=9)$ ASO-treated ob/ob of mice Ucp1, PR/SET Domain 16 (Prdm16), peroxisome proliferator-activated receptor (PPAR) gamma co-activator 1 (Ppargc1a) and PPAR alpha (Ppara) as indicators of thermogenesis and fatty acid $\beta$-oxidation; and PPAR gamma (Pparg) and adiponectin (Adipoq), as indicators of adipocyte differentiation. Results were normalized with Actin (Actb). Values are presented as means \pm SEM for time course representations, and as means \pm SD for histograms. Statistically significant differences between groups are indicated by ${ }^{\star} p<0.05,{ }^{\star \star} p<0.01$, and ${ }^{\star \star \star} p<0.001$ (two-tailed Student's test). Statistical analysis performed by two-way ANOVA test comparing Control ASO HFD vs. Mat7a ASO HFD is presented in GTT and ITT curves. Source data are provided as a Source data file.

demonstrate that targeting Mat1a promotes the secretion of FGF21 in both, DIO and genetically induced obesity (ob/ob mice) models. Our data also show that these increased FGF21 levels originate from hepatocytes and are mediated by Mat1a.

The hepatokine FGF21 has a potential therapeutic effect in the treatment of obesity. Animal studies using a FGF21 analog ${ }^{35}$ or overexpression of FGF $21^{36}$, have demonstrated the ability to reverse obesity and related co-morbidities such as insulin resistance, adiposity and NAFLD. In humans, serum FGF21 levels are increased in patients with NAFLD ${ }^{37}$, and recent studies have demonstrated the potential beneficial effects of FGF21 in NASH. In animal models of NASH, treatment with FGF21 reduces lipotoxicity and ameliorates liver injury ${ }^{38}$ while mice deficient in FGF21 are more prone to develop NAFLD on an obesogenic diet $^{39}$. In a recent clinical trial, treatment with a FGF21 analog, Pegbelfermin, reduces the hepatic fat fraction, as measured by MRI, in patients with $\mathrm{NASH}^{40}$. Our results here confirm that targeting Mat1a protects the liver from the obesity-induced hepatosteatosis through the formation of FGF21. The antisteatotic role of Mat1a inhibition has been an unexpected finding as downregulation of Mat1a has always been associated with NAFLD and cancer development ${ }^{6}$. Indeed, patients with liver cirrhosis show low Mat1a levels and lower production of SAMe ${ }^{41}$. Moreover, Mat1a-KO mice spontaneously develop NAFLD with age; NASH at 8-month-old, which progresses to hepatocellular carcinoma (HCC) at 16-month-old ${ }^{6,42}$. Interestingly, in the context of obesity, we show here that Mat1a deficiency, rather than worsening the liver status, decreased the de novo lipogenesis in the liver, without affecting FAO, preventing lipid accumulation without producing liver fibrosis or infiltration. The same phenotype was observed when Mat1a-KO mice were treated with a HFD. We found that targeting Mat1a, induced BAT thermogenesis, in a mechanism in which FGF21-regulated signaling mTORC1/S6K is involved, as has been described before ${ }^{20}$. Targeting Mat1a in lean mice fed a CD, also increased serum FGF21 levels but not to levels found in HFD-fed mice. However, it was not linked to changes in liver FAO, as in HFD-fed mice, it did not induce changes in body weight or energy expenditure.

In this context of obesity, with high increased serum FGF21 levels, dietary lipids, fatty acids released by lipolysis of the WAT and those secreted by the liver into VLDL were mainly moved towards the BAT to be catabolized through FAO. This mechanism will promote the decrease in serum lipids, as was previously reported by Schlein et al. ${ }^{43}$, as a role of FGF21. The transport of lipids towards the BAT along with the decreased de novo lipogenesis are mechanisms involved in protecting the liver from hepatosteatosis. A role for FGF21 in modulation of de novo lipogenesis in liver has been reported before; $\mathrm{Xu}$ et al. ${ }^{44}$ found that the antisteatotic effect of FGF21 is linked to the inhibition of the nuclear sterol regulatory element binding protein-1 (SREBP-1) and the expression of a variety of genes involved in fatty acid and triglyceride synthesis. The fact that in the livers of Mat1a ASO-treated HFD-fed mice the levels of key enzymes involved in de novo lipogenesis, FAS and ACC, were highly decreased, together with the de novo lipogenesis flux, supports the hypothesis that FGF21 might be inhibiting SREBP1c also in this model. However, gene expression of Acaca, Acacb and Fasn remained unchanged suggesting that a mechanism regulating protein stability should be involved. Thus, our results confirm the beneficial effect of FGF21, which not only protects from obesity, insulin resistance, and dyslipidemia but also prevents NAFLD, as shown in DIO mice with FGF21 deficiency in hepatocytes, where the Mat1a ASO treatment increased liver TG to higher levels than in the ASO-treated DIO control mice.

A transcription factor that has been described as a regulator of FGF21 is NRF2 ${ }^{45}$, which is responsible for the antioxidant response program in the cell. It also confers resistance to various environmental stressors and plays a role in organism metabolic homeostasis $^{45}$. The results here demonstrate that targeting Mat1a in DIO mice increased activation of NRF2 and that inhibiting NRF2 in Mat1a ASO-treated DIO mice hepatocytes reduced FGF21 secretion to normal values. Thus, in this context of obesity, the results suggest that deficiency of Mat1a induces the secretion of FGF21 through activation of NRF2.

Activation of NRF2 results in beneficial effects similar to those obtained with dietary MR; NRF2 activators prevent HFD-induced obesity and adiposity ${ }^{46}$, increased energy expenditure, reduced blood glucose, insulin, and plasma lipid levels 47 , and reduce liver lipid accumulation in DIO and diabetic mice ${ }^{46,47}$. However, as our results here showed, when HFD-fed mice were treated with the Mat1a ASO, the obtained phenotype was driven by FGF21 and not NRF2.

NRF2 is inactivated by Kelch-like ECH-associated protein (KEAP)1, a protein that binds it and acts as a substrate adaptor for the Cullin-3-containing E3 (CUL3-RBX1) ubiquitin ligase, which ubiquitinizes NRF2 and promotes its degradation in the proteasome ${ }^{45}$. Factors such as oxidative stress oxidizes KEAP1 cysteine residues, promoting a conformational change that leaves free NRF2, which is translocated directly into the nucleus ${ }^{45,48}$. Our results showed that the Mat1a ASO treatment in HFD-fed mice reduced the GSH levels in liver and increased lipid peroxidation, which suggest that mitochondria ROS might be elevated as it has been described in Mat1a-KO hepatocytes ${ }^{27}$. Moreover, the addition of GSH or the antioxidant NAC to hepatocytes from 

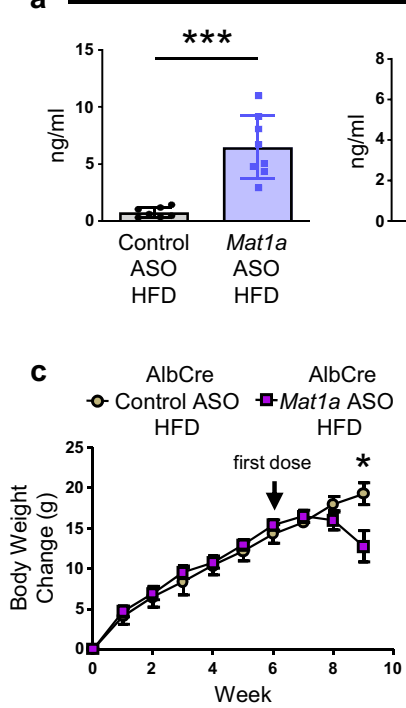

e

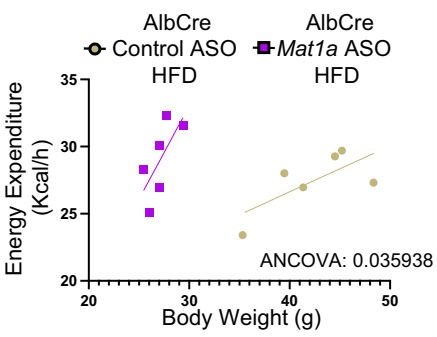

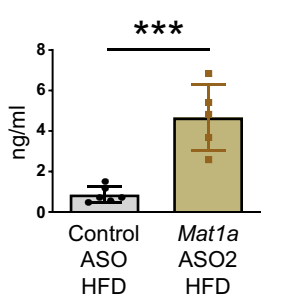
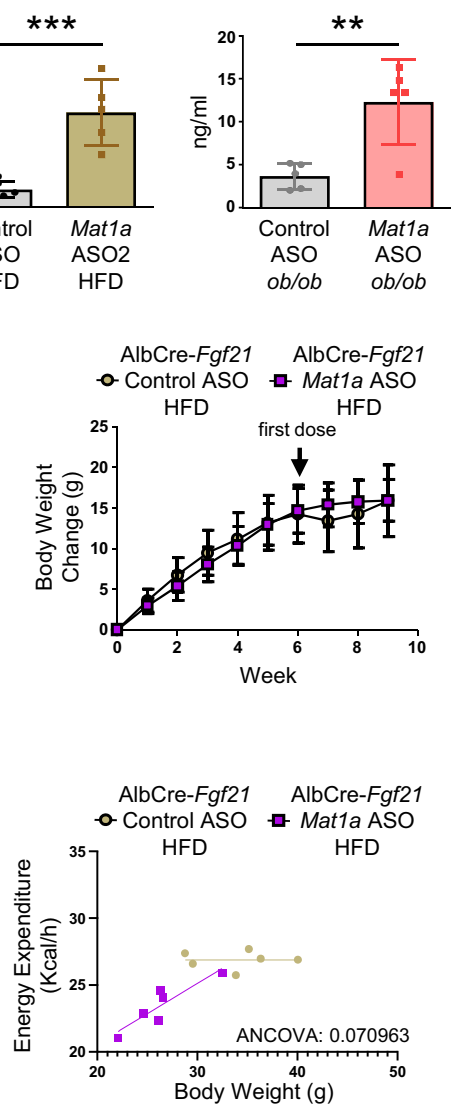

FGF21 Secreted

by hepatocytes

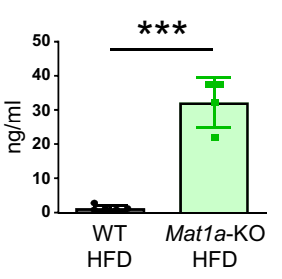

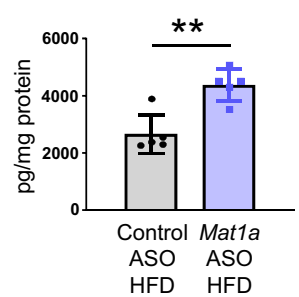

Serum FGF-21

d

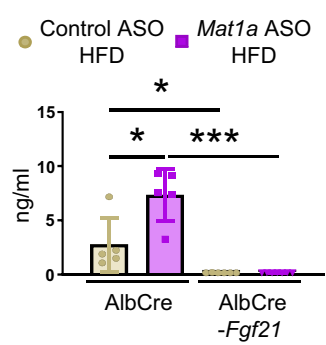

f
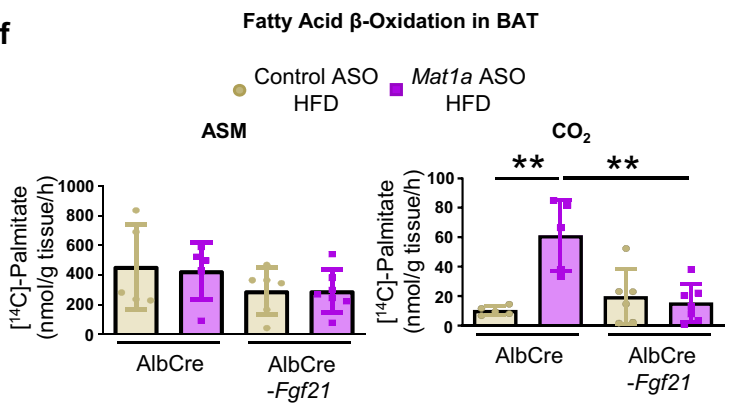

BAT
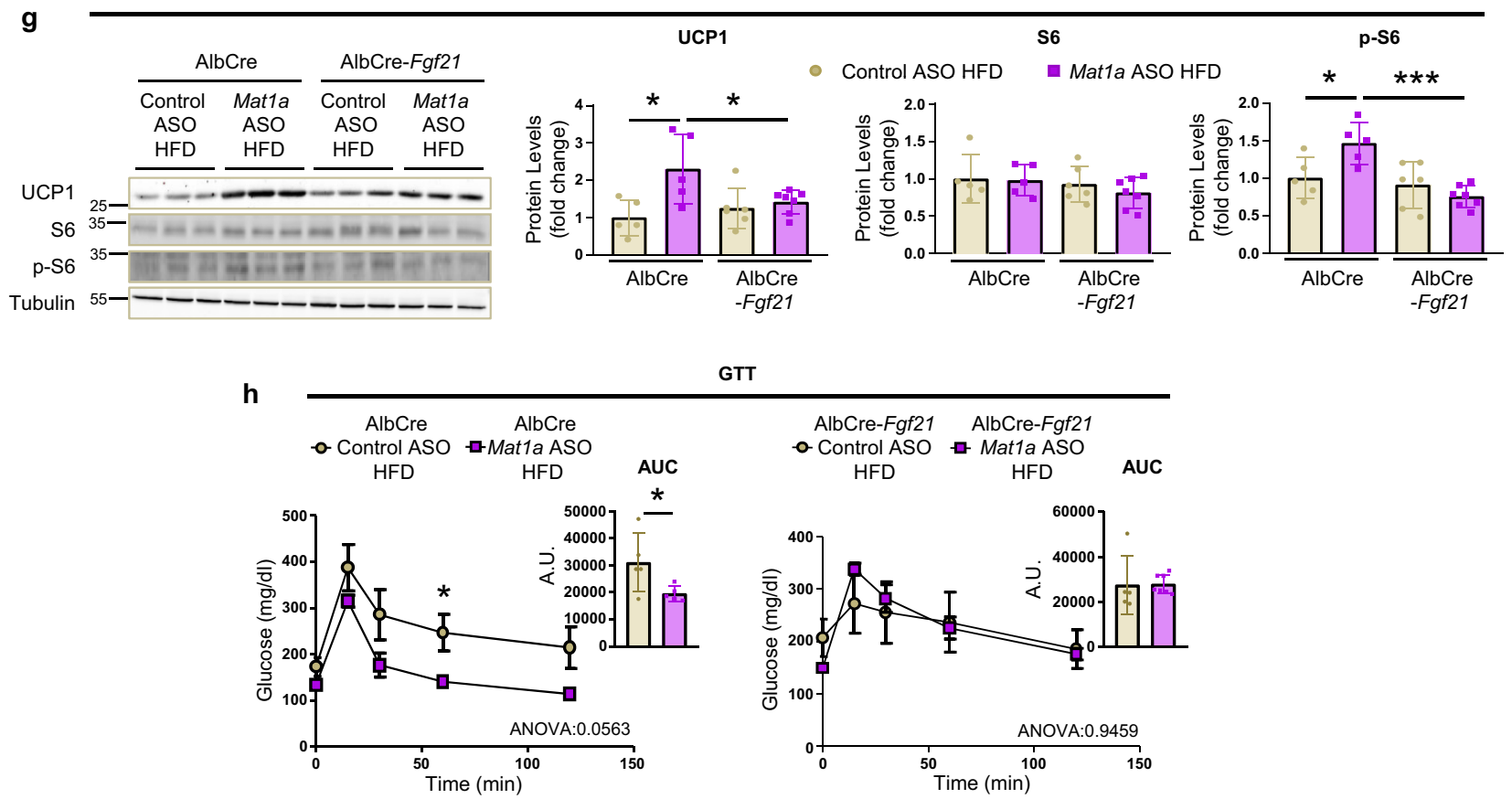

Mat1a ASO-treated HFD-fed mice, restored the secretion of FGF21. Thus, the increased ROS in Mat1a ASO-treated mice might inhibit the KEAP1-NRF2 link, releasing NRF2 and allowing its translocation into the nucleus, binding to the Fof21 promoter and protecting the liver from some deleterious effects driven by Mat1a deficiency. The results suggest that the deficiency of SAMe, probably together with other factors related to Mat1a deficiency, contributes to the increased translocation of NRF2 to the nucleus. It has been recently reported that MATa1 negatively regulates cytochrome P450 2E1 (CYP2E1) expression and that Mat1a knockdown-mediated increase in mitochondrial ROS is CYP2E1 dependent ${ }^{27}$. Thus, our results suggest that 
Fig. 6 Mat1a deficiency induces hepatocyte secretion of fibroblast growth factor 21 (FGF21). Two-month-old C57BL/6J, WT, Mat1a-KO, AlbCre and AlbCre-Fgf21, and 3-month-old ob/ob mice were fed a high-fat diet (HFD) for 4 (ob/ob) or 10 weeks (rest). Last 4 weeks mice were treated with Mat1a antisense oligonucleotides (ASO) (25 mg/kg/week), Mat1a ASO2 (50 mg/kg/week) or control ASO (25 and $50 \mathrm{mg} / \mathrm{kg} /$ week). Hepatocytes pooled from three HFD-fed ASO-treated C57BL/6J mice were seeded (75,000 cells/well) and incubated for $24 \mathrm{~h}$. a Serum FGF21 levels from HFD-fed control ( $n=7$ ) and Mat1a $(n=8)$ ASO-treated C57BL/6J and HFD-fed control $(n=5)$ and Mat1a $(n=5)$ ASO-treated ob/ob mice; control $(n=6)$ and Mat1a ASO2 $(n=5)$-treated C57BL/6J mice; and WT $(n=5)$ and Mat1a-KO $(n=4)$ mice. b FGF21 secreted by hepatocytes $(n=5 /$ group $)$ from HFD-fed ASO-treated mice. c Body weight change for HFD-fed control $(n=5)$ and Mat7a $(n=5)$ ASO-treated AlbCre and control $(n=6)$ and Mat1a $(n=7)$ ASO-treated AlbCre-Fgf21 mice. d Serum FGF21 in HFD-fed control $(n=5)$ and Mat1a $(n=5)$ ASO-treated AlbCre and control $(n=6)$ and Mat1a $(n=7)$ ASO-treated AlbCre-Fgf21 mice. e Energy balance of HFD-fed control ( $n=6 /$ group) and Mat1a ( $n=6 /$ group) ASO-treated AlbCre and AlbCre-Fgf21 mice. $\mathbf{f}$ BAT fatty acid $\beta$-oxidation, as $\left[{ }^{14} \mathrm{C}\right]$-acid-soluble metabolites (ASM) and $\left[{ }^{14} \mathrm{C}\right]-\mathrm{CO}_{2}$ in HFD-fed control $(n=5)$ and Mat7a $(n=5)$ ASO-treated AlbCre and control $(n=6)$ and Mat1a $(n=7)$ ASO-treated AlbCre-Fgf21 mice. $\mathbf{g}$ Representative blots and densitometries of uncoupling protein1 (UCP1), total and phosphorylated protein S6 and Tubulin, as loading control, in BAT of HFD-fed control $(n=5)$ and Mat7a $(n=5)$ ASO-treated AlbCre and control $(n=6)$ and Mat1a $(n=7)$ ASO-treated AlbCre-Fgf21 mice. h Glucose tolerance test (GTT) for HFD-fed control $(n=5)$ and Mat1a $(n=5)$ ASO-treated AlbCre and control $(n=5)$ and Mat1a $(n=7)$ ASO-treated AlbCre-Fgf21 mice. Data are also indicated as area under the curve (AUC). Values are means \pm SEM for time-courses, and means \pm SD for histograms. Statistically significant differences are indicated by ${ }^{\star} p<0.05,{ }^{\star \star} p<0.01$, and ${ }^{{ }^{\star \star \star}} p<0.001$ (two-tailed Student's test). Statistical analysis in GTT is performed by two-way ANOVA test comparing Control ASO HFD vs. Mat7a ASO HFD. Statistical analysis for energy expenditure is performed by two-way ANCOVA test. Source data are provided as a Source data file.

activation of NRF2 might also be directly mediated by Mat1a deficiency, through the cooperation with CYP2E1.

Finally, we propose that silencing Mat1a in obesity activates NRF2 in hepatocytes and induces the secretion of FGF21 to the general circulation, which increases WAT lipolysis and BAT thermogenesis, decreasing de novo lipogenesis in the liver. The movement of circulating lipids towards the BAT, to be catabolized, will reduce circulating lipids in the bloodstream (Fig. 8). In conclusion, targeting Mat1a prevents and reverses obesity and the obesity-related insulin resistance and hepatosteatosis.

\section{Methods}

Animals and housing conditions. 10-week-old male C57BL/6J mice and C57BL/6J liver-specific fibroblast growth factor 21 ( Fgf21) knockout (AlbCre-Fgf21) and 12week-old male B6.Cg-Lep ${ }^{o b}(o b / o b)$ mice were used for Mat1a gene knockdown in the liver. 10-week-old Mat1a knockout (Mat1a-KO) male mice were also included.

Mice were fed a rodent chow diet (Teklad Global 18\% Protein Rodent Diet 2018S; Envigo INC., USA) or a high-fat diet (HFD) (60\% fat calories, Bioserv. F3282) during 10 weeks. For $o b / o b$ mice, HFD treatment was maintained during the weeks of the ASO treatments. Mice body weight and food intake were measured weekly. All mice were housed in a temperature of $21-22{ }^{\circ} \mathrm{C}$ and $40 \%$ humiditycontrolled room, with a $12 \mathrm{~h}$-light/dark cycle and ad libitum access to food and water. Animal procedures were approved by the Ethics Committee for Animal Welfare of the University of the Basque Country UPV/EHU (CEEA 401/2015), CIMUS, University of Santiago de Compostela-Instituto de Investigación Sanitaria (15010/17/007) and Centro Nacional de Investigaciones Cardiovasculares (CNIC) (PROEX 215/18) and were conducted in conformity with the EU Directives for animal experimentation.

\section{In vivo assays}

Matla ASO treatment. For Mat1a knockdown, two different antisense oligonucleotides (ASO), provided by IONIS pharmaceuticals (USA), were used. Mice fed a HFD for 10 weeks were injected intraperitoneal (IP) $25 \mathrm{mg} / \mathrm{kg} /$ week of Mat $1 \mathrm{a}$ Gen 2.0 ASO (5'-CCACTTGTCATCACTCTGGT-3') or control ASO (5'-CCTTCCCT GAAGGTTCCTCC-3'), or 50 mg/kg/week of Mat 1 a Gen 2.0 ASO2 (5'-GCTCAG GAGACATTGACCAT-3') or control ASO (mentioned above), in a single dose from the sixth week of the diet. Mice were sacrificed $48 \mathrm{~h}$ after the last dose.

Long-term treatments and Matla downregulation. 10-week-old male C57BL/6J mice were fed a HFD for 16 weeks. During the last 9 weeks, mice were injected intraperitoneally (IP) $25 \mathrm{mg} / \mathrm{kg} /$ week of Mat 1a ASO or control ASO, in a single dose, until the mice weight loss was stabilized. Mice were sacrificed $48 \mathrm{~h}$ after the last dose.

Lentivirus vector production and administration. Lentiviruses were produced as previously described ${ }^{49}$ with some modifications. Human embryonic kidney (HEK)293 T cells were plated at 25-35\% confluence in Dulbecco's Modified Eagle Medium (DMEM) (Gibco) supplemented with 10\% fetal bovine serum (FBS) (Sigma), $200 \mathrm{mM}$ L-glutamine (Lonza), and 10,000 U/ml penicillin/streptomycin (P/S) (1:1, Lonza). Transient calcium phosphate co-transfection of HEK-293T cells was done with the pGIPZ empty vector or short hairpin RNAs (shRNAs) against UCP1
(pGIPZ.UCP1 vector, V2LMM_51198, Dharmacon) and $\beta$-klotho (pGIPZ.Klb vector, V2LMM_10501, Dharmacon), together with $\mathrm{p} \Delta 8.9$ and $\mathrm{pVSV}-\mathrm{G}$ packaging plasmids. The supernatants containing the lentiviral particles were collected $48 \mathrm{~h}$ after removal of the calcium phosphate precipitate and were filtered through 0.45 $\mu \mathrm{m}$ filters and concentrated by ultracentrifugation for $2 \mathrm{~h}$ at $115,500 \times g$ at $4{ }^{\circ} \mathrm{C}$ (Ultra-Clear Tubes, SW28 rotor and Optima L-100 XP Ultracentrifuge; Beckman Coulter). Viruses were resuspended in cold sterile phosphate-buffered saline (PBS) and mice were bilaterally injected in the BAT under sevoflurane anesthetics in a volume of $100 \mu$ lentiviral particles as previously described ${ }^{50}$.

Corporal and interscapular temperature measurement. Body temperature was detected by a rectal probe connected to a digital thermometer (AZ 8851K/J/T Handheld Digital Thermometer-Single, AZ Instruments Corp., Taiwan $)^{51}$. BAT interscapular temperature was visualized and quantified by thermographic images using a FLIR T430sc Infrared Camera (FLIR Systems, Inc., Wilsonville, OR) and analyzed through Flir R-Tools specific software package ${ }^{51}$.

Indirect calorimetric system. For $48 \mathrm{~h}$, at the end of the ASO treatment, animals were analyzed for Energy Expenditure (EE, kcal/h), Respiratory Quotient (RQ, $\mathrm{VCO}_{2} / \mathrm{VO}_{2}$ ) and Locomotor Activity (LA) using an indirect calorimetric system (LabMaster; TSE Systems; Bad Homburg, Germany) as described previously ${ }^{52}$. The system is an open-circuit instrument that determines the energy consumed by the amount of caloric intake (kilocalories) along time (h); the ratio between the $\mathrm{CO}_{2}$ production and $\mathrm{O}_{2}$ consumption $\left(\mathrm{VCO}_{2} / \mathrm{VO}_{2}\right)$; and the total horizontal locomotion, measured as beam breaks. Previously, all mice were acclimated to the experimental room and habituated to the system for $48 \mathrm{~h}$ before starting the measurements.

To test the $\beta$-adrenergic stimulation sensitivity in mice, $\mathrm{VO}_{2}$ consumption was measured after a $\beta 3$ adrenergic agonist treatment using the indirect calorimetric system. For that purpose, animals were acclimated to the room for $4 \mathrm{~h}$, and then were treated with a single intraperitoneal dose of the $\beta 3$ adrenergic agonist CL316243 in a dose of $1 \mathrm{mg} / \mathrm{kg}^{53}$. The relationship between $\mathrm{VO}_{2}$ consumption and mice weight was analyzed 30 and $45 \mathrm{~min}$ after $\beta$-adrenergic agonist administration using an ANCOVA test.

Insulin and glucose tolerance test. Insulin tolerance tests (ITT) were performed following a $4 \mathrm{~h}$ fasting by IP injection of $0.75 \mathrm{U} / \mathrm{kg}$ insulin. Blood glucose measurements were taken using a Contour Ultra blood glucometer before and 15, 30, 60 and $120 \mathrm{~min}$ after injection of insulin. Glucose tolerance tests (GTT) were performed by oral gavage of glucose at a dose of $1.5 \mathrm{~g} / \mathrm{kg}$ after overnight fasting. Blood glucose was measured before and 15, 30, 60 and $120 \mathrm{~min}$ after glucose administration. In addition, during the GTT, serum insulin levels were measured before and 30,60, and 120 min after oral glucose administration using a commercially available ELISA kit.

Hepatic triglyceride (TG) secretion rate. Mice were subjected to an IP injection with Pluronic F-127 (Poloxamer P-407, Invitrogen Life Technologies, USA) at $1 \mathrm{~g} / \mathrm{kg}$ after an overnight fasting 54 . Prior to injection, 2 and $4 \mathrm{~h}$ after, blood samples were collected, serum prepared, and TG concentrations determined. Commercially available kits were used to measure TG levels (A. Menarini Diagnostics, Spain).

Fat tolerance test. Fat tolerance test was performed with $10 \mu \mathrm{l} / \mathrm{g}$ body weight of olive oil by oral gavage after overnight fasting ${ }^{16}$. Circulating TG levels were measured in plasma prior to oral gavage and 1,2,3, and $4 \mathrm{~h}$ after oil administration using a commercially available kit (A. Menarini Diagnostics, Spain). 
a

FGF21 secreted by hepatocytes

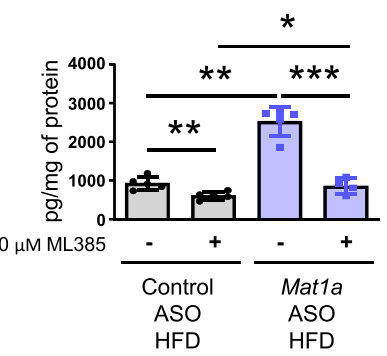

c

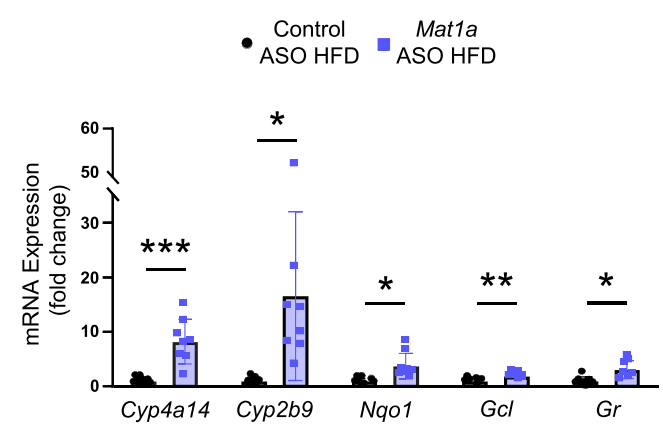

f

Liver TG Concentration

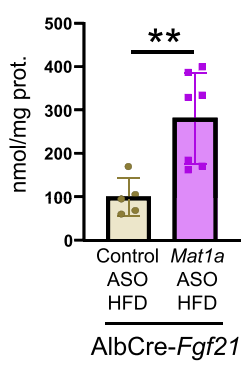

b

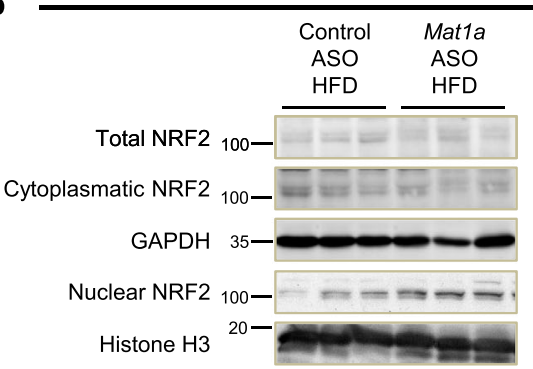

Liver NRF2

Control
ASO HFD ASO HFD

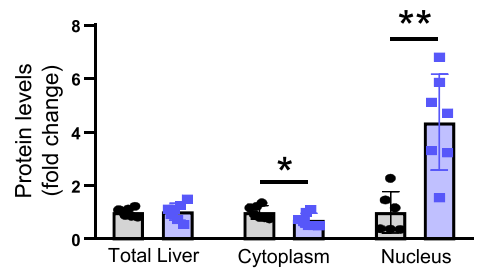

NRF2 ChIP

(hepatocytes)

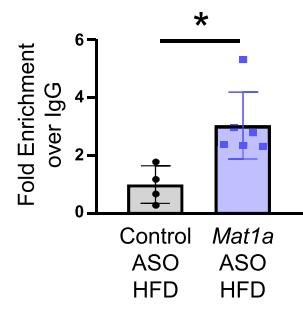

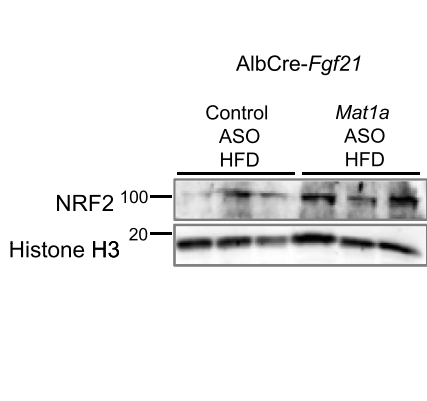

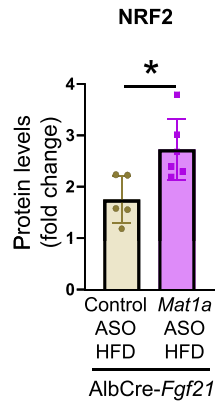

Control Mat1a

- ASO HFD ASO HFD

Glutathione

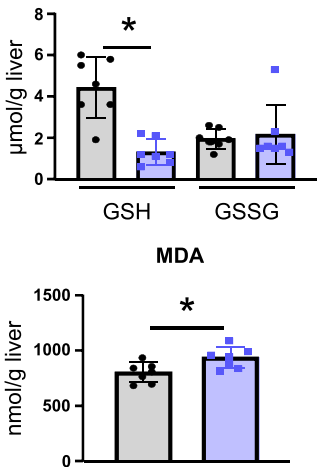

h

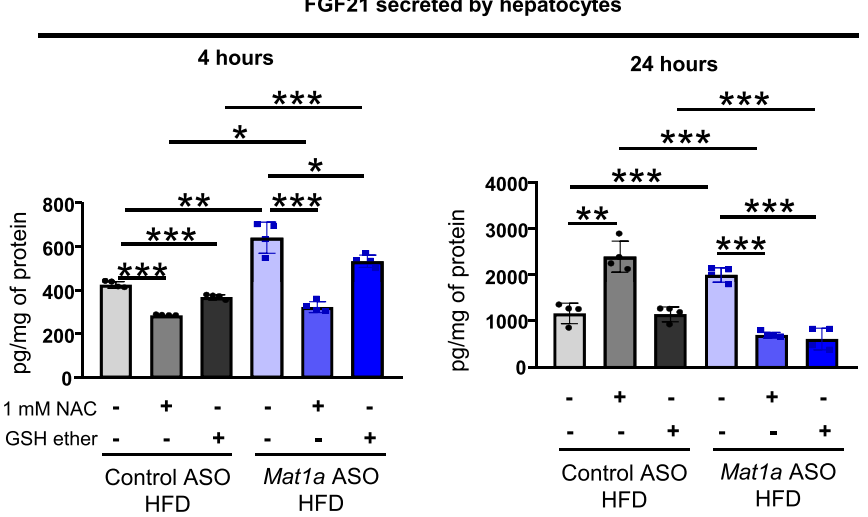

FGF21 secreted by hepatocytes

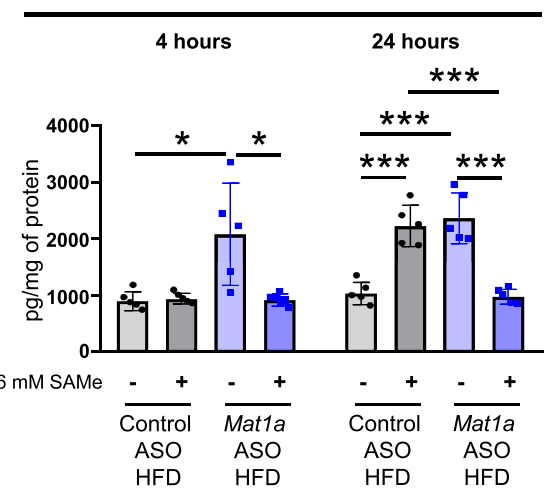

Tissue lipid uptake. Mice were fasted for $4 \mathrm{~h}$ and subjected to oral gavage with $10 \mu \mathrm{l} /$ g body weight of olive oil emulsion containing $2 \mu \mathrm{Ci}\left[{ }^{3} \mathrm{H}\right]$-Triolein (Perkin Elmer INC, USA). Liver, epididymal white adipose tissue (WAT), brown adipose tissue (BAT), muscle and heart were harvested after $4 \mathrm{~h}$. In all, 30-50 mg pieces of each tissue were weight and homogenized in PBS. Homogenate $\left[{ }^{3} \mathrm{H}\right]$-radioactivity was measured with a scintillation counter (Tri-Carb 2810 TR, PerkinElmer, USA) ${ }^{16,55}$.
Tissue and serum extraction. For serum extraction, blood was drawn from cava vein and was allowed to clot at room temperature (RT) for $30 \mathrm{~min}$, then it was cen trifuged at $2000 \times g$ for $30 \mathrm{~min}$ at $4^{\circ} \mathrm{C}$ and the supernatant was centrifuged at $10,000 \times g$ for $10 \mathrm{~min}$ at $4^{\circ} \mathrm{C}$.

Liver, epididymal WAT, BAT, muscle and heart, were collected and washed in fresh cold PBS. Then, tissues were immediately split for metabolic assays, 
Fig. 7 Nuclear factor E2-related factor 2 (NRF2) mediates Mat1a antisense oligonucleotide-induced FGF21 hepatocyte secretion. Two-month-old C57BL/6J mice were fed a high-fat diet (HFD) for 10 weeks. The last 4 weeks mice were treated with Mat7a antisense oligonucleotide (ASO) or control ASO ( $25 \mathrm{mg} / \mathrm{kg} /$ week) until sacrifice. Hepatocytes pooled from three HFD-fed ASO-treated mice were isolated, seeded (106 or 75,000 cells/well) and cultured for 4 or $24 \mathrm{~h}$. a FGF21 secreted by hepatocytes from HFD-fed ASO-treated mice exposed during $24 \mathrm{~h}$ to $10 \mu \mathrm{M}$ of the NRF2 inhibitor ML385 $(n=5)$ or vehicle $(n=5)$ for control ASO and NRF2 inhibitor ML385 $(n=4)$ or vehicle $(n=5)$ for Mat1a ASO. b Representative blots, and densitometries of total liver, cytoplasmic and nuclear NRF2 and glyceraldehyde-3-phosphate dehydrogenase (GAPDH) and Histone H3 from HFD-fed control ( $n=7$, 7, and 6) and Mat1a ( $n=7,7$, and 7) ASO-treated mice. c NRF2 target genes cytochrome P450 4A14 (Cyp4a14), cytochrome P450 2B9 (Cyp2b9), NAD(P)H dehydrogenase [quinone] 1 ( $\mathrm{Nqo1}$ ) (Control $(n=7)$ and Mat1a $(n=8)$ ASO HFD), Glutamate-cysteine ligase (Gcl) and Glutathione reductase (Gr) (Control $(n=6)$ and Mat7a $(n=7)$ ASO HFD) mRNA expression, normalized with Gapdh. d ChIP-q-PCR analysis of NRF2 target gene Fgf21 in hepatocytes from HFD-fed control $(n=4)$ and Mat7a $(n=6)$ ASO-treated mice. e Representative blots, and densitometry of nuclear NRF2 and Histone H3 in liver of HFD-fed control $(n=5)$ and Mat1a $(n=6)$ ASO-treated AlbCre-Fgf21 mice. $\mathbf{f}$ Liver triglyceride (TG) content of HFD-fed control $(n=5)$ and Mat1a $(n=7)$ ASOtreated AlbCre-Fgf21 mice. $\mathbf{g}$ Methionine cycle and transulfuration pathway scheme. Liver reduced (GSH) and oxidized (GSSG) glutathione and malondialdehyde (MDA) levels in HFD-fed control $(n=7)$ and Mat7a $(n=7)$ ASO-treated mice. $\mathbf{h}$ and $\mathbf{i}$ FGF21 secreted by hepatocytes $(n=4$ and $5 /$ group, respectively) from HFD-fed ASO-treated mice exposed during 4 and $24 \mathrm{~h}$ to $\mathbf{h} 5 \mathrm{mM}$ of GSH ether, $1 \mathrm{mM}$ of $\mathrm{N}$-acetylcysteine (NAC) or vehicle; and $\mathbf{i}$ to $6 \mathrm{mM}$ of S-adenosylmethionine (SAMe) or vehicle. Values are represented as means \pm SD. Statistically significant differences between groups are indicated by ${ }^{\star} p<0.05,{ }^{\star \star} p<0.01$, and ${ }^{\star \star \star} p<0.001$ (two-tailed Student's test). Source data are provided as a Source data file.

histological analysis, or to be frozen in liquid nitrogen and stored at $-80^{\circ} \mathrm{C}$ until biochemical analyses were performed.

\section{Metabolic assays}

Fatty acid oxidation. The fatty acid B-oxidation (FAO) was assessed as described before ${ }^{56-59}$. In all, $30 \mathrm{mg}$ of freshly isolated liver and BAT and $60 \mathrm{mg}$ of WAT pieces were homogenated in cold homogenization buffer and sonicated for $10 \mathrm{~s}$. Then, the homogenates were centrifuged at $420 \times g$ for $10 \mathrm{~min}$ at $4{ }^{\circ} \mathrm{C}$ and the supernatant was collected. Approximately $500 \mu \mathrm{g}$ of protein from the liver and BAT homogenates and $250 \mu \mathrm{g}$ of protein from WAT homogenates were used for the assay in a volume of $60 \mu \mathrm{l}$. The reaction was started adding $340 \mu \mathrm{l}$ of assay buffer containing $0.5 \mu \mathrm{Ci} / \mathrm{ml}$ [1-14 C]-Palmitic acid to the samples, and was incubated for $30 \mathrm{~min}$ at $37^{\circ} \mathrm{C}$ in eppendorf tubes with a Whatman paper circle in the cap. The reaction was stopped by adding $200 \mu \mathrm{l}$ of $1 \mathrm{M}$ perchloric acid, after adding $1 \mathrm{M}$ $\mathrm{NaOH}$ in the Whatman cap to collect all the evaporated $\left[{ }^{14} \mathrm{C}\right]-\mathrm{CO}_{2}$.

After $1 \mathrm{~h}$, the Whatman caps were retired, and the radioactivity associated was measured in a scillation counter. The eppendorf tubes were centrifugated at $21,000 \times g$ for $10 \mathrm{~min}$ at $4{ }^{\circ} \mathrm{C}$ and $400 \mu \mathrm{l}$ from the supernatant were collected to measure the radioactivity incorporated in acid-soluble metabolites (ASM).

Measurements of oxygen consumption rate. The respiration of BAT mitochondria was measured at $37^{\circ} \mathrm{C}$ by high-resolution respirometry with the Seahorse Bioscience XF24-3 Extracellular Flux Analyzer, as described before ${ }^{60}$. For the measurement of the oxygen consumption rate (OCR), as the rate change of dissolved $\mathrm{O}_{2}$, BAT mitochondria were isolated by a method similar to Schnaitman and Greenawalt ${ }^{61}$ and basal respiration measurements were made in the presence of succinate and rotenone. The normalized data were expressed as pmol of $\mathrm{O}_{2}$ per minute or milli- $\mathrm{pH}$ units $(\mathrm{mpH})$ per minute, per $\mu \mathrm{g}$ mitochondrial protein.

De novo lipogenesis. For de novo lipogenesis fluxes, $40 \mathrm{mg}$ liver pieces were incubated with the lipogenesis assay medium during $4 \mathrm{~h}$. Then, liver pieces and media were collected. To assess the $\left[{ }^{3} \mathrm{H}\right]$-acetate radioactivity incorporated into TGs, lipids were extracted and separated by thin layer chromatography (TLC) as described before ${ }^{62}$. Lipid species were developed with iodine vapor, scratched, and the silica containing the TGs was introduced in vials with the scintillation liquid. The radioactivity was determined in a scintillation counter.

Adipose tissue lipolysis. Fresh WAT and BAT pieces were incubated and after $4 \mathrm{~h}$, media were collected and the secreted glycerol and fatty acids were measured with commercial available kits (Wako Chemicals, USA, for fatty acids; Sigma-Aldrich, USA, for glycerol) ${ }^{16}$.

\section{Histochemistries}

Hematoxylin and eosin staining. Liver, WAT, and BAT pieces were fixed in $10 \%(\mathrm{v} / \mathrm{v})$ non-buffered formalin for $24 \mathrm{~h}$ at $4{ }^{\circ} \mathrm{C}$ and were kept in $50 \%(\mathrm{v} / \mathrm{v})$ ethanol until they were paraffinized. The paraffin blocks were prepared and cut in $5 \mu \mathrm{m}$-thick sections, and sections were subjected to conventional hematoxylin and eosin staining. Briefly, sections were submerged for $2.5 \mathrm{~min}$ in Shandon TM Harris hematoxylin (Thermo Scientific; USA), washed in water for $5 \mathrm{~min}$, decolorized by immersion in $0.5 \%(\mathrm{v} / \mathrm{v})$ $\mathrm{HCl}$ and washed with $\mathrm{dH}_{2} \mathrm{O}$. Then samples were counterstained with Eosin- $\mathrm{Y}$ Alcoholic (Thermo Scientific, USA) for $25 \mathrm{~s}$, washed and dehydrated with increasing ethanol solutions. stained in eosin for $15 \mathrm{~min}$. Finally, samples were mounted using DPX mounting medium. Representative micrographs were taken under 20x objective from upleft optical microscope.
Sirius red. For the evaluation of liver fibrosis, liver pieces were fixed in $10 \%(\mathrm{v} / \mathrm{v})$ non-buffered formalin (Sigma-Aldrich, USA) for $24 \mathrm{~h}$ at $4{ }^{\circ} \mathrm{C}$ and were kept in $50 \%$ $(\mathrm{v} / \mathrm{v})$ ethanol until they were paraffinized. The paraffin blocks were prepared and cut in $5 \mu \mathrm{m}$-thick sections with the microtome and stained with Sirius red solution $(0.01 \%(w / v)$ Fast Green FCF/0.1\% (w/v) Sirius red in picric acid (Sigma-Aldrich; USA) for $30 \mathrm{~min}$. Sections were then dehydrated directly in $100 \%$ alcohol and mounted in DPX mounting media. Representative micrographs were taken under 20x objective from upright optical microscope. Stained area percentage of each sample were calculated using FRIDA 1.0 software (FRamework for Image Dataset Analysis, Johns Hopkins University, USA).

Sudan Red. For the histological evaluation of lipid storage in liver, Sudan Red staining was performed. OCT frozen livers were cut in $8 \mu \mathrm{m}$-thick sections and fixed with $10 \%(\mathrm{v} / \mathrm{v})$ non-buffered formalin for $2 \mathrm{~min}$. Then samples were incubated with freshly prepared Sudan III stain (Sigma-Aldrich; USA) and counterstained with Mayers Hematoxylin (Sigma-Aldrich; USA). Finally, they were mounted with aqueous mounting media. Representative micrographs were taken under 20x objective. Percentage of stained area of each sample was calculated using FRIDA 1.0 software (Framework for Image Dataset Analysis, http:// bui3.win.ad.jhu.edu/frida/, Johns Hopkins University; USA).

\section{Immunoassays}

Immunohistochemical analysis of F4/80. For the evaluation of liver inflammation, $\mathrm{F} 4 / 80$ immunostaining was performed. OCT frozen livers were cut in $8 \mu \mathrm{m}$-thick sections, unmasked according to the primary antibody to be used and subjected to peroxide blocking, $3 \%(\mathrm{v} / \mathrm{v}) \mathrm{H}_{2} \mathrm{O}_{2}$ in PBS, during $10 \mathrm{~min}$ at RT. For stainings, samples were blocked with goat anti-mouse FAB fragment (Jackson Immunoresearch; USA), and blocked with $5 \%(\mathrm{v} / \mathrm{v})$ goat serum. Then, sections were incubated with the primary antibody in DAKO antibody diluent in a 1:50 dilution during $1 \mathrm{~h}$ at $37^{\circ} \mathrm{C}$, followed by Envision anti-rabbit or anti-mouse (DAKO; Denmark) HRP-conjugated secondary antibody incubation. Colorimetric detections were confirmed with vector VIP chromogen (Vector; USA) and sections were counterstained with hematoxylin. Samples were mounted using DPX mounting medium. For the analysis, images were taken with an upright light microscope. Representative micrographs were taken under 20x objective. Stained area percentage of each sample were calculated using FRIDA 1.0 software.

Western blotting. The liver, BAT, and WAT tissues were homogenized with the homogenization buffer. In some cases, when required, liver nucleus were extracted using a subcellular proteome extraction kit (Calbiochem, Germany) following the manufacturer's instructions. The protein lysates were subjected to sodium dodecyl sulphate-polyacrylamide gel electrophoresis, electrotransferred and immobilized onto nitrocellulose membranes, and incubated with commercial primary antibody. For protein detection, membranes were incubated with a horseradish peroxidase (HRP) or a fluorescent dye containing secondary antibody. Fluorescent dye-linked secondary antibody bound proteins were detected by fluorescent excitation and emission in the fluorescent detection system Chemidoc (Bio-Rad, USA). For quantification, Quantity One 29.0 and ImageLab 6.0.1 softwares (Bio-Rad, USA) were used, and the antibodies that were used are detailed in Supplementary Table 7.

Chromatin immunoprecipitation. Chromatin immunoprecipitation (ChIP) analysis in isolated hepatocytes were performed as describe before ${ }^{58}$. Briefly, after hepatocytes isolation and attachment, crosslinking were directly performed on the culture plates by addition of formaldehyde to a $1 \%$ final concentration. Crosslinking was stopped with glycine to $0.125 \mathrm{M}$ final concentration. Next, cell pellets were collected, lysated and chromatin was sonicated on a Diagenode Bioruptor to 


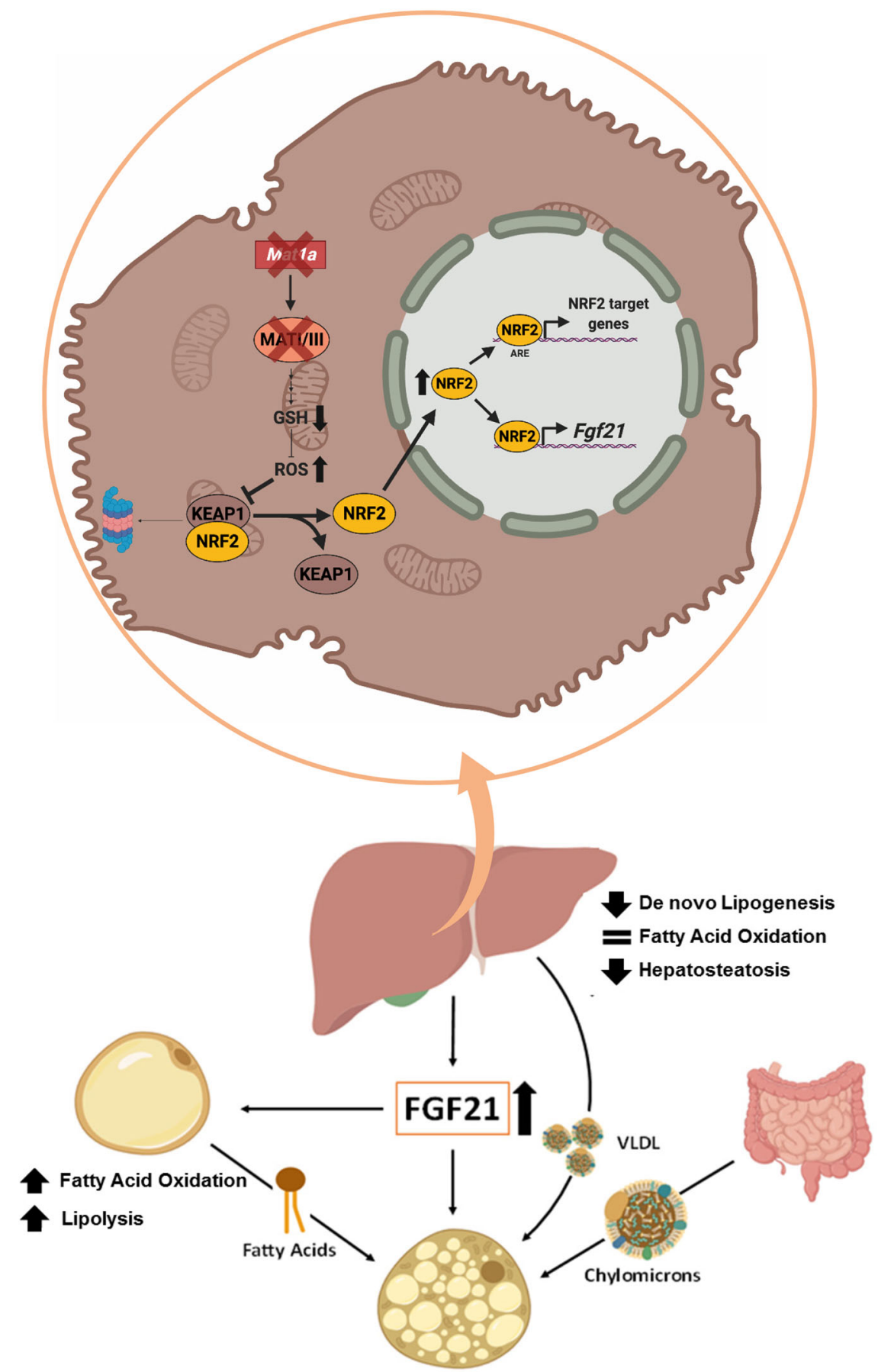

Thermogenesis

1. Fatty Acid Oxidation

- Linolvsis

Fig. 8 Proposed model of reversion of obesity and obesity-related hepatosteatosis by Mat1a antisense oligonucleotides. Mat1a deficiency leads to a reduction in glutathione levels, leading to ROS accumulation in hepatocytes. The increased ROS levels modifies KEAP1, triggering NRF2 release to the nucleus. NRF2 induces transcription of target genes including FGF21. The increased FGF21 expression and secretion to the circulation induces BAT thermogenesis, lipolysis and release of fatty acids from white adipose tissue (WAT) to the blood. Lipids released to plasma as very-low-density lipoproteins (VLDL), free fatty acids and dietary lipids (chylomicrons) are channeled to the BAT to be used as energy source in the fatty acid oxidation. The increased FGF21 also reduces liver triglyceride storage and de novo lipogenesis. Thus, the NRF2-induced FGF21 secretion reverses obesity protecting from insulin resistance, hepatosteatosis and reducing plasma lipid levels. This picture was designed using BioRender graphic tool (BioRender.com).

an average length of 200-500 bp. Inmunoprecipitation of crosslinked-Protein/DNA complexes was achieved by using protein $\mathrm{G}$ magnetic beads (ThermoFischer), which were incubated at $4^{\circ} \mathrm{C}$ for $3 \mathrm{~h}$ in a rotation mixer with anti-NRF2 as antibody of interest and normal mouse IgG as negative control (see Supplementary Table 7 for antibodies). Complexes were eluted and reverse crosslinking was performed. The DNA obtained was extracted and purified with Qiagen QIAquick PCR purification kit and quantification of immunoprecipitate-enriched DNA sequences was performed by real-time PCR. PCR primers were designed for the already described NRF2 binding site in the Fof 21 regulatory region ${ }^{63}$, which was confirmed considering the INSECT 2.0 predicted binding sites for NRF2 
in the regulatory region of Fgf21. Primers sequences are detailed in Supplementary Table 8.

ELISA tests. Serum FGF21 (FGF21 Quantikine ELISA kit, Cat. No. MF2100, R\&D Systems), Insulin (Ultra Sensitive Mouse Insulin ELISA Kit, Crystal Chem, Cat. No. 90080) epinephrine/adrenaline (Epinephrine/Adrenaline ELISA kit, Cat. No. CSBE08679m, Cusabio), noradrenaline (ELISA Kit for Noradrenaline, Cat. No ABK1E1601, Abyntek), adiponectin (Adiponectin ELISA Kit, Cat. No. ab108785, Abcam), apolipoprotein C2 (Apolipoprotein CII ELISA kit, Cat. No. CSBEL001932MO, Cusabio) and apolipoprotein C3 (Apolipoprotein CIII ELISA Kit, Cat. No. ab217777, Abcam) levels were quantified using commercially available ELISA kits according to the manufacturers' protocols.

\section{Quantification of biochemical parameters}

Quantification of TGs in liver. Pieces of livers $(30 \mathrm{mg})$ were homogenized in 10 volumes of ice-cold saline buffer. Lipids were extracted following the Folch method ${ }^{64}$ and dissolved in isopropanol. TG quantification was performed with a commercial kit (A. Menarini Diagnostics, Spain) following manufacturer's instructions.

Serum measurements. Serum ALT levels were measured using a commercially available kit (Randox, UK) following manufacturer's instructions. Serum TG and fatty acid (FA) levels in fasted and fed conditions were measured using commercially available kits (A. Menarini Diagnosis, Spain and Wako Chemicals, USA respectively) following manufacturer's instructions.

Lipid peroxidation assay kit. Liver malondialdehyde (MDA) content, used as a marker for lipid peroxidation, was quantified using a commercially available kit (Sigma-Aldrich, USA) following manufacturer's instructions.

Quantification of glutathione. Liver reduced glutathione (GSH) and oxidized glutathione (GSSG) levels were quantified using a commercially available kit (Abcam, USA) following manufacturer's instructions.

RNA isolation and quantitative PCR. RNA was extracted using Trizol Reagent (Invitrogen, Spain) and cDNAs were obtained by retrotranscription (SuperScript III $\mathrm{RT}$, Invitrogen, USA) following the manufacturers' instructions. Real-Time qPCRs were performed using SYBR ${ }^{\circledast}$ Green Supermix. All reactions were performed in duplicate, and expression levels were normalized to the average level of Gapdh and $A c t b$ genes in each sample using the geNorm 3.1 software. The oligonucleotides and their sequences used for qPCR analysis are collected in the Supplementary Table 8.

\section{In vitro experiments}

Hepatocyte Isolation. Hepatocytes from Mat1a ASO and control ASO-treated HFD-fed mice were isolated. For this, perfusion with collagenase type I was used as described previously ${ }^{65}$. In brief, animals were anesthetized with IP injection of sodium pentobarbital (Euthasol) $(150 \mathrm{mg} / \mathrm{kg}$ of body weight), the abdomen was opened, and a catheter was inserted into the inferior vena cava while the portal vein was cut. Next, liver was washed by perfusion with Krebs-Henseleit $(\mathrm{KH})$ perfusion medium equilibrated with fizzed carbogen at $37^{\circ} \mathrm{C}$. After the washing, EGTA $0.05 \%(\mathrm{w} / \mathrm{v})$ was added to the $\mathrm{KH}$ medium and the perfusion was maintained for $5 \mathrm{~min}$. Finally, an enzymatic digestion was performed during $10-12 \mathrm{~min}$ with $\mathrm{KH}$ perfusion medium supplemented with $\mathrm{Ca}^{2+}, 300 \mu \mathrm{g} / \mathrm{ml}$ collagenase and $60 \mu \mathrm{g} / \mathrm{ml}$ trypsin inhibitor (Roche, Switzerland). After perfusion, the liver was gently disaggregated. The viable cells were purified by density centrifugation at $40 \times g$ for $2 \mathrm{~min}$ at RT. Isolated pure hepatocytes were seeded over collagen and fibronectincoated culture dishes (24-well dishes) at a density of $75 \times 10^{5}$ cells/well in the medium for cell adhesion. Cells were placed at $37^{\circ} \mathrm{C}$ in a humidified atmosphere of $5 \% \mathrm{CO}_{2}-95 \%$ air

Cultures of hepatocytes. Isolated hepatocytes were suspended in serum-free Dulbecco's modified Eagle's medium (DMEM). After $2 \mathrm{~h}$ of attachment, $75 \times 10^{5}$ cells/ well in 24-well dishes were incubated with or without SAMe $(6 \mathrm{mM})$, methioninedeficient medium, the protein kinase RNA-like endoplasmic reticulum kinase (PERK) inhibitor GSK2606414 $(2 \mu \mathrm{M})$, the PPARa inhibitor GW6471 $(100 \mu \mathrm{M})$, $\mathrm{N}$-acetylcysteine $(1 \mathrm{mM})$ and reduced glutathione $(\mathrm{GSH})$ ester $(5 \mathrm{mM})$ and the NRF2 inhibitor ML385 $(10 \mu \mathrm{M})$. ML385 is a small molecule that binds to NRF2 and inhibits its downstream target gene expression. Specifically, ML385 binds and inhibits to the Neh1, the Cap "N" Collar Basic Leucine Zipper (CNC-bZIP) domain of NRF2, interfering with the binding of the V-Maf Avian Musculoaponeurotic Fibrosarcoma Oncogene Homolog G (MAFG)-NRF2 protein complex to regulatory DNA binding sequences ${ }^{66}$. Cultured hepatocytes were also treated with gene silencing small interfering RNAs (siRNAs) for Nrf2, Atf4, and Ppara. After the incubation period, media were collected and FGF21 levels were measured using a commercially available ELISA kit. The amount of FGF21 secreted by hepatocytes was normalized by the $\mathrm{mg}$ of the hepatocyte total protein.

Brown adipose tissue (BAT) primary cell isolation and culture. For primary adipocyte cultures, stromal vascular cells were obtained from interscapular BAT excised from
HFD-fed ASO-treated C57BL/6J mice as described by Oeckl et al. ${ }^{67}$, with minor modifications. Briefly, for brown adipocyte differentiation, confluent precursor cells were cultured with DMEM, supplemented with $10 \%$ fetal bovine serum (v/v), $40 \mu \mathrm{g} /$ $\mathrm{ml}$ gentamicin and penicillin/streptomycin mixture, $850 \mathrm{nM}$ insulin, $1 \mathrm{nM}$ triiodothyronine (T3), and $1 \mu \mathrm{M}$ rosiglitazone. Cells were used 8 days later, when more than $90 \%$ of the cells were differentiated, showing fat depots. For brown adipocyte lipolysis, fatty acid $\beta$-oxidation and FGF21 secretion, confluent precursor cells were induced for 2 days in differentiation media supplemented with $1 \mu \mathrm{M}$ dexamethasone, $500 \mu \mathrm{M} 3$-isobutyl-1-methylxanthine (IBMX) and $125 \mu \mathrm{M}$ indomethacin and differentiated for the next 6 days, when more than $90 \%$ of the cells were differentiated.

Statistical analysis. Multiple independent experiments were conducted to verify the reproducibility of the data. For this study 310 mice have been used, from those 293 mice were treated with the ASOs (152 with control ASO, 126 with Mat1a ASO, and 15 with Mat1a ASO2), the rest were Mat1a-KO mice and their controls. Experiments in vivo were repeated at least ten times and the in vitro experiments were repeated at least three times. All attempts to replicate experiments were successful.

Data are represented mean \pm SEM or mean \pm SD as detailed in each figure. Differences between groups were tested using the two-tailed Student's $t$-test and two-way ANOVA. The relationship between Energy expenditure and body weight, and $\mathrm{VO}_{2}$ consumption and body weight was tested with two-way ANCOVA68. Significance was defined as $p \leq 0.05$. These analyses were performed using GraphPad Prism 8.0 and Excel software 2016.

Reporting summary. Further information on research design is available in the Nature Research Reporting Summary linked to this article.

\section{Data availability}

No data sets with mandated depositions are presented in the study. The authors declare that the data supporting the findings of this study are available within the paper and its supplementary information files. The data generated in this study are provided in the supplementary information/Source data file. Source data are provided with this paper.

Received: 24 July 2020; Accepted: 3 February 2022;

Published online: 01 March 2022

\section{References}

1. Blüher, M. Obesity: global epidemiology and pathogenesis. Nat. Rev. Endocrinol. 15, 288-298 (2019).

2. Polyzos, S. A., Kountouras, J. \& Mantzoros, C. S. Obesity and nonalcoholic fatty liver disease: from pathophysiology to therapeutics. Metabolism 92, 82-97 (2019)

3. Boland, M. L. et al. Resolution of NASH and hepatic fibrosis by the GLP-1R/ GcgR dual-agonist Cotadutide via modulating mitochondrial function and lipogenesis. Nat. Metab. 2, 413-431 (2020).

4. Elshorbagy, A. K. et al. S-adenosylmethionine is associated with fat mass and truncal adiposity in older adults. J. Nutr. 143, 1982-1988 (2013).

5. Elshorbagy, A. K., Jernerén, F., Samocha-Bonet, D., Refsum, H. \& Heilbronn, L. K. Serum S-adenosylmethionine, but not methionine, increases in response to overfeeding in humans. Nutr. Diabetes 6, 2-5 (2016).

6. Lu, S. C. et al. Methionine adenosyltransferase 1A knockout mice are predisposed to liver injury and exhibit increased expression of genes involved in proliferation. Proc. Natl Acad. Sci. USA 98, 5560-5565 (2001).

7. Virtanen, J. K. et al. High dietary methionine intake increases the risk of acute coronary events in middle-aged men. Nutr. Metab. Cardiovasc. Dis. 16, 113-120 (2006).

8. Elshorbagy, A. K. et al. Cysteine supplementation reverses methionine restriction effects on rat adiposity: significance of stearoyl-coenzyme A desaturase. J. Lipid Res. 52, 104-112 (2011).

9. Doshi, S. et al. Relationship between S-adenosylmethionine, Sadenosylhomocysteine, asymmetric dimethylarginine, and endothelial function in healthy human subjects during experimental hyper- and hypohomocysteinemia. Metab. Clin. Exp. 54, 351-360 (2005).

10. Miller, R. A. et al. Methionine-deficient diet extends mouse lifespan, slows immune and lens aging, alters glucose, T4, IGF-I and insulin levels, and increases hepatocyte MIF levels and stress resistance. Aging Cell 4, 119-125 (2005).

11. Malloy, V. L. et al. Methionine restriction decreases visceral fat mass and preserves insulin action in aging male Fischer 344 rats independent of energy restriction. Aging Cell 5, 305-314 (2006).

12. Jacobs, R. L. et al. Impaired de novo choline synthesis explains why phosphatidylethanolamine N-methyltransferase-deficient mice are protected from diet-induced obesity. J. Biol. Chem. 285, 22403-22413 (2010).

13. Wan, S. et al. Hepatic PEMT activity mediates liver health, weight gain, and insulin resistance. FASEB J. 33, 10986-10995 (2019). 
14. Hong, S. et al. Nicotinamide $\mathrm{N}$-methyltransferase regulates hepatic nutrient metabolism through Sirtl protein stabilization. Nat. Med. 21, 887-894 (2015).

15. Kraus, D. et al. Nicotinamide $\mathrm{N}$-methyltransferase knockdown protects against diet-induced obesity. Nature 508, 258-262 (2014).

16. Martínez-Uña, M. et al. Excess S-adenosylmethionine reroutes phosphatidylethanolamine towards phosphatidylcholine and triglyceride synthesis. Hepatology 58, 1296-1305 (2013).

17. Martínez-Chantar, M. L. et al. Loss of the glycine N-methyltransferase gene leads to steatosis and hepatocellular carcinoma in mice. Hepatology 47 , 1191-1199 (2008).

18. Murray, B. et al. Structure and function study of the complex that synthesizes S-adenosylmethionine. IUCrJ 1, 240-249 (2014).

19. Cano, A. et al. Methionine adenosyltransferase $1 \mathrm{~A}$ gene deletion disrupts hepatic very low-density lipoprotein assembly in mice. Hepatology $\mathbf{5 4}$, 1975-1986 (2011).

20. Minard, A. Y. et al. mTORC1 is a major regulatory node in the FGF21 signaling network in adipocytes. Cell Rep. 17, 29-36 (2016).

21. Romero-Gómez, M., Zelber-Sagi, S. \& Trenell, M. Treatment of NAFLD with diet, physical activity and exercise. J. Hepatol. 67, 829-846 (2017).

22. Anstee, Q. M. \& Goldin, R. D. Mouse models in non-alcoholic fatty liver disease and steatohepatitis research. Int. J. Exp. Pathol. 87, 1-16 (2006).

23. Stone, K. P., Wanders, D., Orgeron, M., Cortez, C. C. \& Gettys, T. W. Mechanisms of increased in vivo insulin sensitivity by dietary methionine restriction in mice. Diabetes 63, 3721-3733 (2014).

24. Badman, M. K. et al. Hepatic fibroblast growth factor 21 is regulated by PPARalpha and is a key mediator of hepatic lipid metabolism in ketotic states. Cell Metab. 5, 426-437 (2007).

25. Salminen, A., Kaarniranta, K. \& Kauppinen, A. Regulation of longevity by FGF21: Interaction between energy metabolism and stress responses. Ageing Res. Rev. 37, 79-93 (2017).

26. Furusawa, Y., Uruno, A., Yagishita, Y., Higashi, C. \& Yamamoto, M. Nrf2 induces fibroblast growth factor 21 in diabetic mice. Genes Cells 19, 864-878 (2014).

27. Murray, B. et al. Methionine adenosyltransferase $\alpha 1$ is targeted to the mitochondrial matrix and interacts with cytochrome P450 2E1 to lower its expression. Hepatology 70, 2018-2034 (2019).

28. Younossi, Z. M. et al. Global epidemiology of nonalcoholic fatty liver diseaseMeta-analytic assessment of prevalence, incidence, and outcomes. Hepatology 64, 73-84 (2016).

29. Chobot, A., Górowska-Kowolik, K., Sokołowska, M. \& Jarosz-Chobot, P. Obesity and diabetes - not only a simple link between two epidemics. Diabetes Metab. Res. Rev. 34, 1-9 (2018).

30. Chan, J. C. N. et al. The central roles of obesity-associated dyslipidaemia, endothelial activation and cytokines in the Metabolic Syndrome-an analysis by structural equation modelling. Int. J. Obes. 26, 994-1008 (2002).

31. Elshorbagy, A. K., KoziC, H. V., David Smith, A. \& Refsum, H. Cysteine and obesity: consistency of the evidence across epidemiologic, animal and cellular studies. Curr. Opin. Clin. Nutr. Metab. Care 15, 49-57 (2012).

32. Yu, D. et al. Short-Term methionine deprivation improves metabolic health via sexually dimorphic, mTORC1-independent mechanisms. FASEB J. 32, 3471-3482 (2018).

33. Malloy, V. L. et al. Methionine restriction prevents the progression of hepatic steatosis in leptin-deficient obese mice. Metabolism 62, 1651-1661 (2013).

34. Wanders, D. et al. FGF21 mediates the thermogenic and insulin-sensitizing effects of dietary methionine restriction but not its effects on hepatic lipid metabolism. Diabetes 66, 858-867 (2017).

35. Gaich, G. et al. The effects of LY2405319, an FGF21 analog, in obese human subjects with type 2 diabetes. Cell Metab. 18, 333-340 (2013).

36. Jimenez, V. et al. FGF21 gene therapy as treatment for obesity and insulin resistance. EMBO Mol. Med. 10, 1-24 (2018).

37. Li, H. et al. Fibroblast growth factor 21 levels are increased in nonalcoholic fatty liver disease patients and are correlated with hepatic triglyceride. $J$. Hepatol. 53, 934-940 (2010).

38. Fisher, F. M. et al. Fibroblast growth factor 21 limits lipotoxicity by promoting hepatic fatty acid activation in mice on methionine and choline-deficient diets. Gastroenterology 147, 1073-1083.e6 (2014).

39. Singhal, G. et al. Deficiency of fibroblast growth factor 21 (FGF21) promotes hepatocellular carcinoma (HCC) in mice on a long term obesogenic diet. Mol. Metab. 13, 56-66 (2018).

40. Sanyal, A. et al. Pegbelfermin (BMS-986036), a PEGylated fibroblast growth factor 21 analogue, in patients with non-alcoholic steatohepatitis: a randomised, double-blind, placebo-controlled, phase 2a trial. Lancet 392, 2705-2717 (2018).

41. Mato, J., Alvarez, L., Ortiz, P. \& Pajares, M. A. S-adenosylmethionine synthesis: molecular mechanisms and clinical implications. Pharm. Ther. 73, 265-280 (1997).

42. Martínez-Chantar, M. L. et al. Spontaneous oxidative stress and liver tumors in mice lacking methionine adenosyltransferase 1A. FASEB J. 16, 1292-1294 (2002).
43. Schlein, C. et al. FGF21 lowers plasma triglycerides by accelerating lipoprotein catabolism in white and brown adipose tissues. Cell Metab. 23, 441-453 (2016).

44. Xu, J. et al. Fibroblast growth factor 21 reverses hepatic steatosis, increases energy expenditure, and improves insulin sensitivity in diet-induced obese mice. Diabetes 58, 250-259 (2009).

45. Hayes, J. D. \& Dinkova-Kostova, A. T. The Nrf2 regulatory network provides an interface between redox and intermediary metabolism. Trends Biochem. Sci. 39, 199-218 (2014).

46. Shin, S. et al. Role of Nrf2 in prevention of high-fat diet-induced obesity by synthetic triterpenoid CDDO-imidazolide. Eur. J. Pharm. 620, 138-144 (2009).

47. $\mathrm{Yu}, \mathrm{Z}$. et al. Oltipraz upregulates the nuclear respiratory factor 2 alpha subunit (NRF2) antioxidant system and prevents insulin resistance and obesity induced by a high-fat diet in C57BL/6J mice. Diabetologia 54, 922-934 (2011).

48. Uruno, A. \& Motohashi, H. The Keap1-Nrf2 system as an in vivo sensor for electrophiles. Nitric Oxide 25, 153-160 (2011).

49. González-Terán, B. et al. Eukaryotic elongation factor 2 controls TNF- $\alpha$ translation in LPS-induced hepatitis. J. Clin. Invest. 123, 164-178 (2013).

50. Folgueira, C. et al. Hypothalamic dopamine signaling regulates brown fat thermogenesis. Nat. Metab. 1, 811-829 (2019).

51. Matesanz, N. et al. P38A blocks brown adipose tissue thermogenesis through P38 inhibition. PLoS Biol. 16, 1-29 (2018).

52. Suárez, J. et al. Oleoylethanolamide enhances $\beta$-adrenergic-mediated thermogenesis and white-to-brown adipocyte phenotype in epididymal white adipose tissue in rat. Dis. Model Mech. 7, 129-141 (2014).

53. Yamaguchi, $\mathrm{S}$. et al. Adipose tissue NAD+ biosynthesis is required for regulating adaptive thermogenesis and whole-body energy homeostasis in mice. Proc. Natl Acad. Sci. USA 116, 23822-23828 (2019).

54. Millar, J. S., Cromley, D. A., McCoy, M. G., Rader, D. J. \& Billheimer, J. T. Determining hepatic triglyceride production in mice: comparison of poloxamer 407 with Triton WR-1339. J. Lipid Res. 46, 2023-2028 (2005).

55. Yen, C.-L. E. et al. Deficiency of the intestinal enzyme acyl CoA:monoacylglycerol acyltransferase-2 protects mice from metabolic disorders induced by high-fat feeding. Nat. Med. 15, 442-446 (2009).

56. Hirschey, M. D. et al. SIRT3 regulates mitochondrial fatty-acid oxidation by reversible enzyme deacetylation. Nature 464, 121-125 (2010).

57. Huynh, F. K., Green, M. F., Koves, T. R. \& Hirschey, M. D. Measurement of fatty acid oxidation rates in animal tissues and cell lines. Methods Enzymol. 542, 391-405 (2014).

58. Gonzalez-Romero, F. et al. E2F1 and E2F2-mediated repression of CPT2 establishes a lipid-rich tumor-promoting environment. Cancer Res. 81, 2874-2887 (2021)

59. Barbier-Torres, L. et al. Silencing hepatic MCJ attenuates non-alcoholic fatty liver disease (NAFLD) by increasing mitochondrial fatty acid oxidation. Nat. Commun. 11, 1-15 (2020).

60. Martínez-Sánchez, N. et al. Hypothalamic AMPK-ER stress-JNK1 axis mediates the central actions of thyroid hormones on energy balance. Cell Metab. 26, 212-229.e12 (2017).

61. Schnaitman, C. \& Greenawalt, J. W. Enzymatic properties of the inner and outer membranes of rat liver mitochondria. J. Cell Biol. 38, 158-175 (1968).

62. Gómez-Santos, B. et al. Liver osteopontin is required to prevent the progression of age-related nonalcoholic fatty liver disease. https://doi.org/ 10.1111/acel.13183 (2020)

63. Erickson, A. \& Moreau, R. The regulation of FGF21 gene expression by metabolic factors and nutrients. Horm. Mol. Biol. Clin. Investig. 30, 1-14 (2017).

64. Folch, J., Lees, M. \& Sloane Stanley, G. H. A simple method for the isolation and purification of total lipides from animal tissues. J. Biol. Chem. 226, 497-509 (1957).

65. Smedsrød, B. \& Pertoft, H. Preparation of pure hepatocytes and reticuloendothelial cells in high yield from a single rat liver by means of percoll centrifugation and selective adherence. J. Leukoc. Biol. 38, 213-230 (1985).

66. Liu, F. et al. Small molecule inhibitor of NRF2 selectively intervenes therapeutic resistance in KEAP1-deficient NSCLC tumors. ACS Chem. Biol. 19, 1358-1370 (2018)

67. Oeckl, J., Bast-Habersbrunner, A., Fromme, T., Klingenspor, M. \& Li, Y. Isolation, culture, and functional analysis of murine thermogenic adipocytes. STAR Protoc. 1, 100118 (2020).

68. Tschöp, M. H. et al. A guide to analysis of mouse energy metabolism. Nat. Methods 9, 57-63 (2012).

\section{Acknowledgements}

This work was supported by Ayudas para apoyar grupos de investigación del sistema Universitario Vasco (IT971-16) and MCIU/AEI/FEDER, UE (RTI2018-095134-B-100) (to P.A.), (RTI2018-099413-B-I00 and RED2018-102379-T) (to R.N.), PID2020119486RB-100 (to M.V.R.) and (RTI2018-096759-A-100) (to T.C.D). EFSD/Lilly 
European Diabetes Research Program, MICIU (PID2019-104399RB-I00), Fundación AECC PROYE19047SABI, and Comunidad de Madrid IMMUNOTHERCAN-CM B2017/BMD-3733 (to G.S.). La CAIXA Foundation LCF/PR/HP17/52190004, MINECOFEDER SAF2017-87301-R, AYUDAS FUNDACIÓN BBVA A EQUIPOS DE INVESTIGACIÓN CIENTÍFICA UMBRELLA 2018 and AECC Scientific Foundation, grant name: Rare Cancers 2017 (to M.L.M.-C.). AECC Scientific Foundation (to T.C.D.). Xunta de Galicia 2020-PG015 (to R.N.) Gilead Sciences International Research Scholars Program in Liver Disease (to M.V.R.). Personal fellows: E.P.F. was awarded with Juan de la Cierva-Formación, FJC2018-035449-I. C.F. was awarded with Sara Borrell (CD19/ 00078). CIC bioGUNE thanks MCIU for the Severo Ochoa Excellence Accreditation (SEV-2016-0644). The authors thank Dr. Manuel Lafita's laboratory (Getxo, Bizkaia, Spain) for his valuable help in the analysis of biochemical parameters.

\section{Author contributions}

D.S.U., X.B., and P.A. designed the project; D.S.U., X.B., G.S., M.L.M.-C., R.N., and P.A. designed experimental protocols; D.S.U., X.B., P.O., M.A.-B., A.N.-Z., M.R.G., N.G., C.F., E.M.N., A.M., B.P., V.M.-R., T.C.D., J.L.G.-R., B.G.-S., V.G.J., F.G.-R., I.A., E.P.-F., I.M.-G., M.V.-R., S.B., R.L., J.M.B., W.-K.S., G.S., M.L.M-.C., R.N., and P.A. contributed to investigations and data analysis; D.S.U., X.B., J.L.G.-R., W.-K.S., M.L.M.-C., G.S., R.N., and P.A. contributed to discussions; D.SU., X.B., and P.A. prepared the figures with input from all the other authors, D.S.U. and P.A. wrote the manuscript with input from all the other authors.

\section{Competing interests}

M.L.M.-C. is a consultant of Mitotherapeutix. P.A., X.B., and D.S.U. have a patent application related to this work (EP19762826.6). The remaining authors declare no competing interests.

\section{Additional information}

Supplementary information The online version contains supplementary material available at https://doi.org/10.1038/s41467-022-28749-z.

Correspondence and requests for materials should be addressed to Patricia Aspichueta.

Peer review information Nature Communications thanks the anonymous reviewer(s) for their contribution to the peer review of this work. Peer reviewer reports are available.

Reprints and permission information is available at http://www.nature.com/reprints

Publisher's note Springer Nature remains neutral with regard to jurisdictional claims in published maps and institutional affiliations.

(i) Open Access This article is licensed under a Creative Commons Attribution 4.0 International License, which permits use, sharing, adaptation, distribution and reproduction in any medium or format, as long as you give appropriate credit to the original author(s) and the source, provide a link to the Creative Commons license, and indicate if changes were made. The images or other third party material in this article are included in the article's Creative Commons license, unless indicated otherwise in a credit line to the material. If material is not included in the article's Creative Commons license and your intended use is not permitted by statutory regulation or exceeds the permitted use, you will need to obtain permission directly from the copyright holder. To view a copy of this license, visit http://creativecommons.org/ licenses/by/4.0/.

(c) The Author(s) 2022 